\title{
$N$-茮基脱除研究进展
}

\author{
周光伟张莉珠薛亚涵李加荣* \\ (北京理工大学化学与化工学院 北京 100081)
}

\begin{abstract}
摘要 $N$-茮基是有机胺的常用保护基团，因其脱除具有便捷、高效的特点，在有机合成特别是药物和天然产物研究中 具有重要地位. 近年来, 大量科研工作者对 $N$-苄基脱保护进行广泛的研究, 但相关工作分散, 缺乏系统性综述. 因此, 从还原脱茮、氧化脱苄、酸碱脱苄等几个方面，系统全面地概述了 $N$-苄基脱除研究的进展.
\end{abstract}

关键词 有机胺; $N$-苄基保护; 还原脱芐; 氧化脱苄; 酸碱脱苄

\section{Progress of N-Benzyl Removal}

\author{
Zhou, Guangwei Zhang, Lizhu Xue, Yahan Li, Jiarong* \\ (School of Chemistry and Chemical Engineering, Beijing Institute of Technology, Beijing 100081)
}

\begin{abstract}
N$-Benzyl is a common protecting group for organic amines, owing to its convenient and efficient removal. It plays an important role in organic synthesis, especially in the research of drugs and natural products. In recent years, a large number of researchers have conducted extensive studies on $N$-benzyl deprotection, but the related work is scattered and lacks systematic review. Therefore, the progress of $N$-benzyl removal is systematically and comprehensively summarized from the aspects of reductive, oxidative and acid-base debenzylation.
\end{abstract}

Keywords organic amine; $N$-benzyl protection; reductive debenzylation; oxidative debenzylation; acid-base debenzylation

氨基的保护和脱除是有机合成中常用的策略，在药 物、天然产物等研究中屡见不鲜. 氨基的常见保护基团 主要有三类: 烷氧羰基保护、酰基保护、烷基保护. 芐 基作为一种烷基保护基, 可通过适当的卤代物在碱性条 件下引入. 茮基的脱除也相对容易, 可通过催化氢解、 液氨和钠或锂、氧化脱茮、酸解脱苄等方法脱保护. 除 此之外, 芐基保护的有机胺能在强碱、亲核试剂、有机 金属试剂的存在下保持稳定，因此苄基成为氨基保护最 常用的基团之一 ${ }^{[1 \sim 5]}$. 本文将综述氨基的脱茮研究进展.

\section{1 还原脱苄}

还原脱苄大都采用催化氢解, 即 $N$-芐基的 $\mathrm{C}-\mathrm{N}$ 键 在催化剂作用下发生 $\sigma$-断裂而脱茮. 催化氢解具有反应 条件温和、产率高等优点, 是目前应用最广泛的脱苄方 法. Pd 具有优异的吸氢性能, 是氢解最常用的催化剂, 其组成可以是单质、氧化物、盐等的形式存在. 为了提 高催化性能, Pd 通常负载于多孔材料上, 如活性炭、
$\mathrm{CaCO}_{3} 、 \mathrm{BaSO}_{4}$ 和 $\mathrm{Al}_{2} \mathrm{O}_{3}$ 等 ${ }^{[6,7]}$. 催化氢解根据氢源的不 同, Pd 催化氢解可分为催化氢解和催化氢转移氢解, 前 者是以氢气为氢源, 后者则以甲酸、甲酸铵、环已烯等 为氢源.

\section{1 催化氢解}

催化氢解脱茮是较为常用的脱茮方法, 其过程为氢 气首先被吸附在催化剂上形成原子氢，随后茮基保护的 有机胺与催化剂络合、 $\mathrm{C}-\mathrm{N}$ 键发生断裂、芐基与原子 氢形成甲苯，而胺与原子氢形成脱去苄基的仲胺或伯 胺 ${ }^{[2,5,8,9]}$ (Scheme 1). 对于常见的 $N$-芐基, 催化氢解都能 较好地脱除, 而且对 $O$-芐基、卤素也有一定的脱除效果. 除此之外, 催化氢解以氢气为氢源, 在反应过程中需要 一定的压力, 有时为了加快反应速度还需在加热条件下 进行. 反应温和、产率高是催化氢解脱苄的最大的优势, 但其选择性差、不适用于易被还原的底物是其最主要的 缺点. $\mathrm{Pd} / \mathrm{C}$ 和 $\mathrm{Pd}(\mathrm{OH})_{2} / \mathrm{C}$ 是典型的催化氢解催化剂, 甲 醇、乙醇、乙酸等极性溶剂是适宜的反应溶剂 ${ }^{[10 \sim 16]}$. 此

* Corresponding author. E-mail: jrli@bit.edu.cn

Received January 21, 2019; revised March 13, 2019; published online April 16, 2019.

Project supported by the 086 Special Fund.

086 专项基金资助项目. 
外，酸性条件可促进有机胺质子化，防止催化剂失活， 因此催化氢解脱苄也可在酸性条件下进行.

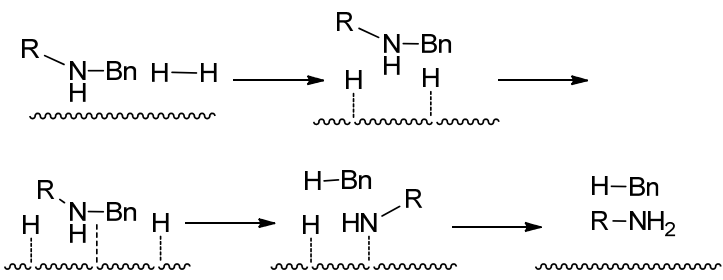

图式 1 催化氢解脱苄反应机理

Scheme 1 Reaction mechanism of catalytic hydrogenolysis and debenzylation

催化氢解在多官能团的氮杂环脱苄研究中优势明 显, Yoshida 等 ${ }^{[17]}$ 使用 $\mathrm{Pd}(\mathrm{OH})_{2} / \mathrm{C}$ 为催化剂在室温下快速 氢解 $N$-茮基胺生成脱苄产物 2 (Eq. 1), 而底物中叔丁基 二甲基硅烷基、四氢吡喃基和三甲基硅烷基等对酸敏感 的基团在该脱芐反应过程中也未被破坏. 反应以强极性 的甲醇作溶剂, 在 $101 \mathrm{kPa}$ 的氢气压力下氢解, 数小时 就能以较高的产率( $81 \%$ \% 99\%)反应完全. 该方法快速、 高效、温和, 尤其适宜于含有敏感基团的底物脱芐.

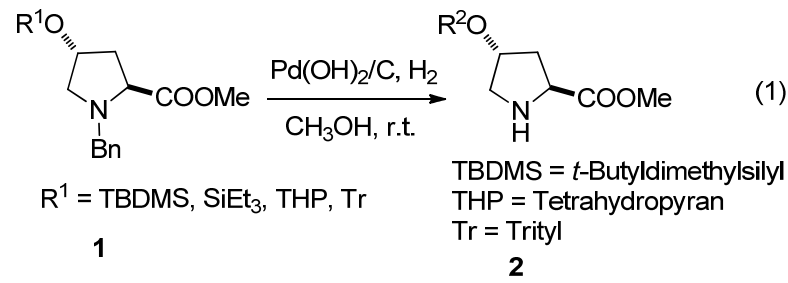

当脱苄产物的稳定性低于反应物时, 简单的催化氢 解往往无法得到脱芐产物, 如高能炸药 CL-20 (HNIW, 六硝基六氮杂异伍兹烷)的制备. CL-20 是目前综合性能 最好的单质炸药, 其合成分为六茮基六氮杂异伍兹烷 (HBIW, 3) 的合成、HBIW 的脱茮、脱芐产物的硝化三 步 ${ }^{[18]}$. HBIW 可通过苄胺和乙二醀缩合制备, 而 HBIW 中苄基的脱保护是 CL-20 成功合成的关键. Bellamy ${ }^{[19]}$ 系统研究了 HBIW 的脱芐情况, 发现在乙酸䣶中以质量 分数为 $20 \% \mathrm{Pd}(\mathrm{OH})_{2} / \mathrm{C}$ 为催化剂氢解 HBIW 可以得到四 乙酰基二苄基异伍兹烷(TADBIW) (Eq. 2), TADBIW 是 制备 HNIW 的重要中间体. 由于异伍兹烷的稳定性要低 于 HBIW, 酸酐的加入能够及时捕获异伍兹烷, 生成酰 基稳定的 TADBIW. 因此, 在 HBIW 脱苄过程中, 使用 强酰化试剂的脱茮效果要好于弱酰化试剂和无酰化试 剂的脱芳效果. 研究还发现, 芐基苯环上无论含有供电 子或吸电子基团, 对脱茮反应均是不利的; 而且湿 Pearlman 催化剂 $\left[\right.$ 质量分数为 $\left.20 \% \mathrm{Pd}(\mathrm{OH})_{2} / \mathrm{C}\right]$ 的催化效 果要优于干 Pearlman 催化剂, 也优于干 Degussa 催化剂 (质量分数为 $10 \% \mathrm{Pd} / \mathrm{C}$ ).

1997 年, Wardle 等 ${ }^{[20]}$ 改进了在酸䣶中催化氢解的条
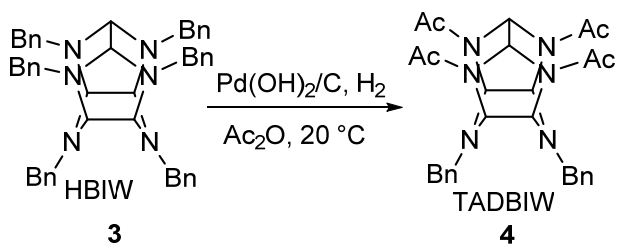

件, 确定了以 $\mathrm{Pd}(\mathrm{OH})_{2} / \mathrm{C}$ 为催化剂, 以二甲基甲酰胺 (DMF)和乙酸䣲组成的混合溶剂的脱茮体系. 在反应中 添加溴化物, 可防止催化剂中毒, 促进催化氢解快速进 行，且大大降低催化剂的使用量(Scheme 2). 目前，大 量关于 HNIW 合成的文献 ${ }^{[21 ~ 24]}$ 均采用 $\mathrm{Pd}(\mathrm{OH})_{2} / \mathrm{C}$ 氢解 的方法, 即首先将 HBIW 催化氢解得到四乙酰基二芐基 异伍兹烷(TADBIW，4), TADBIW 以甲酸为氢供体的条 件下继续氢解得到四乙酰基二甲酰基异伍兹烷(5)和其 他副产物。

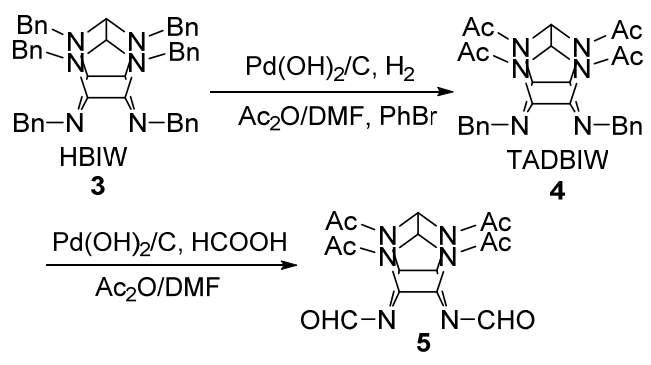

图式 2 六苄基六氮杂异伍兹烷催化氢解脱苠 Scheme 2 Hexabenzylhexaazaisowurtzitane catalytic hydrogenolysis debenzylation

对于催化氢解脱苄，适当向反应体系中添加酸，可 缩短反应时间、降低贵金属催化剂的用量. 2-氨基吡啶 甲基吡咯烷是一类高效选择性神经元型一氧化氮酶抑 制剂，合成这些抑制剂的关键步骤是去除双保护的 2-氨 基吡啶环衍生物 6 中的苄基. 2012 年 $\mathrm{Ji}$ 等 ${ }^{[25]}$ 研究发现, 吡啶环相连氮原子上的苄基脱除是困难的, 以 $\mathrm{Pd} / \mathrm{C}$ 为 催化剂的氢解脱茮和 2,3-二氯-5,6-二氰基对苯醌(DDQ) 为氧化剂氧化脱茮体系均不能脱去 $N$-芐基, 而使用硝 酸铈铵(CAN)的氧化脱茮也仅有 $26 \%$ 的产率. 他们又改 用质量分数为 $20 \% \mathrm{Pd}(\mathrm{OH})_{2} / \mathrm{C}$ 催化剂, 将温度升高到 $60{ }^{\circ} \mathrm{C}$, 并向体系中加入乙酸, 经过一系列改进使得脱 芐产率由最初的 $26 \%$ 提高到 $60 \%$ (Eq. 3). 乙酸的加入促 进吡啶环的质子化，使氢解反应更易进行.

2014 年朱思兰等 ${ }^{[26]}$ 在合成八氢吡咯并 $[3,4-c]$ 吡咯2-碳酸叔丁酯(9)的过程中, 使用 $\mathrm{Pd}(\mathrm{OH})_{2} / \mathrm{C}$ 催化剂脱去 氮原子上的苠基(Eq. 4). 该反应条件温和，以乙醇为溶 剂, 常温常压下即可进行, 同时与氮原子相连的酯也未 被破坏.

氮杂金刚烷是另一种笼型硝铵类含能化合物的骨 架, 罗军等 ${ }^{[27]}$ 利用 $\mathrm{Pd}(\mathrm{OH})_{2} / \mathrm{C}$ 催化氢解方法脱去氮杂金 
<smiles>CCOC(=O)c1ccc2cccc(O[C@@H]3CN(C(=O)OC(C)(C)C)C[C@H]3Cc3cc(C)cc(N(Cc4ccccc4)C(=O)OC(C)(C)C)n3)c2c1</smiles><smiles>Cc1cc(C[C@@H]2CN(C(=O)OC(C)(C)C)C[C@H]2Oc2cccc3ccccc23)nc(NC(C)(C)C)c1</smiles>

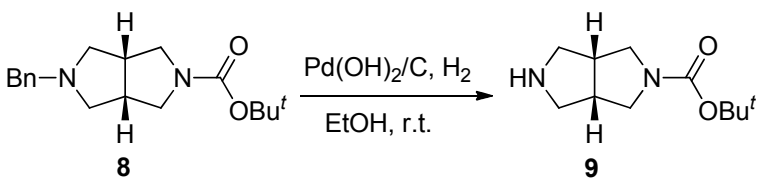

刚烷上的苄基制备了硝基氮杂金刚烷的前体. 由于 $\mathrm{N}$ $\mathrm{H}$ 键较活泼, 脱茮产物在酸性条件下不稳定, 该方法也 以酸酐为溶剂在 $\mathrm{Pd}(\mathrm{OH})_{2} / \mathrm{C}$ 条件下脱茮乙酰化, 得到了 2,4,9-三乙酰基-2,4,9-三氮杂金刚烷- 7-醇(11), 11 在硝硫 混酸中硝化制得 2,4,9-三硝基-2,4,9-三氮杂金刚烷-7-醇 (Eq. 5)

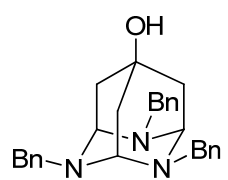

10

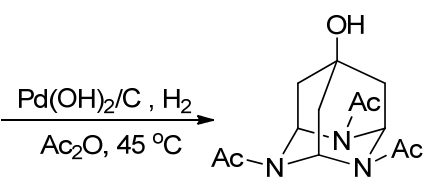

11

$\mathrm{Pd} / \mathrm{C}$ 催化剂是另一种使用广泛的氢解催化剂, 而 溶剂在氢解过程发挥重要的作用, 控制催化氢解的反应 溶剂, 得到意想不到的结果. Bailey 等 ${ }^{[28]}$ 在催化氢解合 成 $(-)$-raumacline (13)过程中, 使用非醇类溶剂, 通常 不反应; 而使用简单醇类溶剂可导致 $N$-烷基化的副反 应发生, 得到的是 (一)-raumacline 和 $N$-烷基化的混合物. 他们改用三氟乙醇作溶剂, 能够达到较高的产率并且无 $N$-烷基化的副产物. 三氟乙醇作溶剂的催化氢解克服了 (一)-raumacline 合成中 $N$-烷基化的问题, 并适用于多种 底物, 令人惊讶的是该方法在 $N$-甲基存在下还可选择 性脱除 $N$-茮基(Eq. 6).
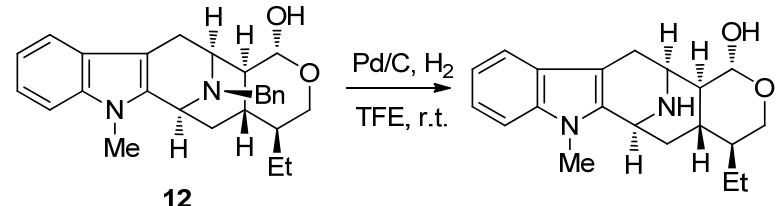

13

传统的催化氢解脱茮具有反应条件温和、产率高等
优点, 但对于低分子量的胺和含多亲水基团有机胺的催 化氢解的产率往往很低，前者是因为低分子量不稳定， 后者是因为其较好的水溶性. Cheng 等 ${ }^{[29]}$ 向催化氢解体 系中引入多氯代烷, 较好地克服了小分子胺的不稳定和 水溶性的问题(Eq. 7). C- $\mathrm{Cl}$ 键会在 $\mathrm{Pd} / \mathrm{C}$ 催化下发生加 氢脱氯协同反应产生 $\mathrm{HCl}$ 和氯代烷，而有机胺作为 $\mathrm{HCl}$ 的受体能加速氢解反应，同时改变多氯代烷的用量可显 著影响氢解反应的速率. 该方法实现了脱苄和成盐两步 合二为一的转化，在不对称合成和天然产物的合成中具 有较好的应用。

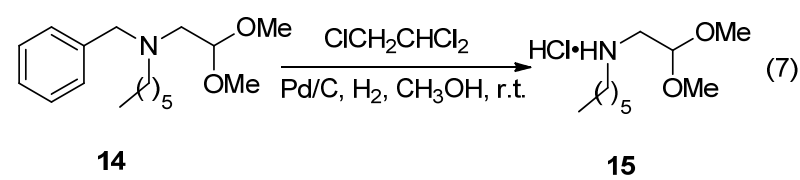

Quast 等 ${ }^{[30]}$ 在研究 2,4-二氮杂二环[3.1.0]己烷-3-氧 (17)的合成和反应活性时, 利用 $\mathrm{Pd} / \mathrm{C}$ 在乙醇中催化氢解 使环丙基开环的同时还能脱去咪唑啉上的两个苄基(Eq. 8). 该催化氢解反应条件温和，在 $98 \mathrm{kPa}$ 压力、 $54{ }^{\circ} \mathrm{C}$ 的 条件下搅拌 $24 \mathrm{~h}$ 就能获得产物.

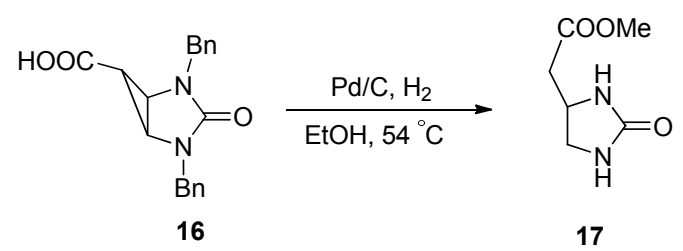

盐酸莫西沙星是一种氟喹诺酮类抗菌药，临床上用 于治疗肺病、肺炎、细菌性鼻窦炎以及皮肤和软组织感 染等疾病. 国内有很多课题组 ${ }^{[31 ~ 34]}$ 在合成盐酸莫西沙 星过程中, 使用 $\mathrm{Pd} / \mathrm{C}$ 催化氢解的方法脱去 $(S, S)-8$-茮基2-甲基-2,8-二氮杂双环 [4.3.0]壬烷(18)中的 $N$-茮基(Eq. 9). 该过程需在 $2.5 \sim 3.0 \mathrm{MPa}$ 的压力下进行，能以超过 $80 \%$ 的产率得到 $(S, S)$-2-甲基-2,8-二氮杂双环[4.3.0]壬烷， 无需纯化即可进行下一步反应. 该脱苄方法虽产率高, 但需在高压釜中进行，对设备提出了一定的要求，是该 脱苠方法的一大缺点.

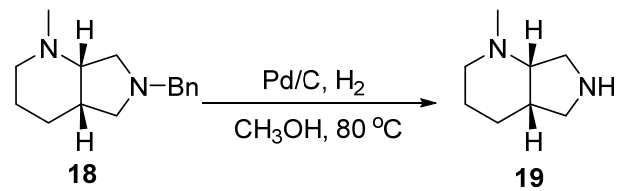

$\mathrm{Pd} / \mathrm{C}$ 作催化剂的脱苄方法不仅适用于小环化合物, 对于含多茮基的大环化合物同样具有较好的脱茮活性. Désogère 等 ${ }^{[35]}$ 使用 $\mathrm{Pd} / \mathrm{C}$ 催化剂成功实现了含三苠基的 九元环化合物 1,4,7-三苠基-1,4,7-三氮杂环壬烷(20)的脱 
芐. 该脱芐反应无需加热, 只在室温、乙酸条件下即可 完成, 并能以 $95 \%$ 高产率得到脱三苄产物 1,4,7-三氮杂 环壬烷(21) (Eq. 10).

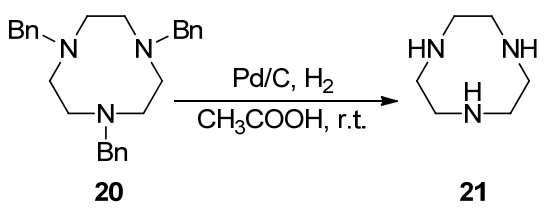

催化脱苄除了适用于链状化合物, 单、双杂环化合 物和对三环化合物如螺桨烷也有较好的脱茮效果. Torres 等 ${ }^{[36]}$ 在研究螺桨烷的抗甲型流感病毒活性时, 合 成了一系列的氮杂螺桨烷. 其中, 对芐基保护的氮杂螺 桨烷脱苄时，先将底物 $\mathbf{2 2}$ 与盐酸反应制成盐酸盐，而后 在甲醇中以 $\mathrm{Pd} / \mathrm{C}$ 催化氢解能高效得到脱苠基的螺桨烷 盐酸盐(Scheme 3). 鉴于螺桨烷盐酸盐的稳定性及氢解 效果明显优于螺桨烷本身, 螺桨烷盐酸盐直接脱苄为不 稳定化合物的脱茮提供了思路.

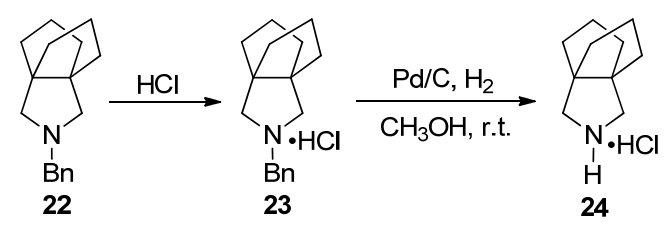

图式 3 [3.3.3]螺桨烷催化氢解脱茮

Scheme 3 [3.3.3]propellane catalytic hydrogenolysis debenzylation

$\mathrm{Pd}(\mathrm{OH})_{2} / \mathrm{C}$ 和 $\mathrm{Pd} / \mathrm{C}$ 是催化氢解两种主要的催化剂, 二种催化剂的混合可达到单一催化剂无法实现的效果. $\mathrm{Li}$ 等 ${ }^{[37]}$ 则报道了用等量 $\mathrm{Pd}(\mathrm{OH})_{2} / \mathrm{C}$ 和 $\mathrm{Pd} / \mathrm{C}$ 混合催化剂 脱茮基的实例, 该体系对于茮基保护的脂肪胺或芳香胺 均具有良好催化效果. 混合催化剂的使用可以大幅缩短 反应的时间, 对单一催化剂不能催化的反应也具有一定 的催化活性.

2018 年, Lou 等 ${ }^{[38]}$ 研究了用双金属催化剂代替单一 的 Pd 金属催化剂制备 HNIW 的反应. 该方法不仅可以 降低贵金属 Pd 的用量, 而且对 HBIW 和 TADBIW 的脱 苠具有选择性. 研究发现, 以沉淀还原的方法制备的 $\mathrm{PdFe}$ 双金属催化剂的催化活性要远高于其他双金属如 $\mathrm{PdNi} 、 \mathrm{PdCu} 、 \mathrm{PdCo}$ 等催化剂. 研究还表明, 以不同载体 所制备的 PdFe 双金属催化剂对 HBIW 和 TADBIW 具有 选择性, 以活性炭为载体的 $\mathrm{PdFe}$ 双金属催化剂只对 TADBIW 有催化活性, 以 $\mathrm{SiO}_{2}$ 和 $\mathrm{Al}_{2} \mathrm{O}_{3}$ 为载体的 $\mathrm{PdFe}$ 双金属催化剂 HBIW 有催化活性, 而以 $\mathrm{TiO}_{2}$ 为载体的 PdFe 双金属催化剂对 HBIW 和 TADBIW 均有脱茮催化 活性.

\section{2 催化氢转移氢解}

催化氢解是以氢气作氢源的非均相催化反应, 因此 为加强氢气在催化剂上的吸附, 在实际使用过程中需在 一定的压力下进行. 催化氢转移氢解作为催化氢解的一 种替代方法, 克服了催化氢解需在压力设备中进行的弊 端. 某些特定化合物在催化剂作用下发生脱氢作用, 氢 原子从供体转移给催化剂, 然后传递给要被氢解的底 物, 以这样的氢源进行的脱苄反应称为氢转移氢 解 ${ }^{[5,8,39]}$. Pd 同样是应用最广泛的催化剂活性成分, 甲 酸 $^{[40,41] \text { 、甲酸铵 }}{ }^{[42 \sim 44]}$ 、环己烯 ${ }^{[45]}$ 和 1,4 -环已二烯 ${ }^{[46]}$ 等可 作为氢供体. 催化氢转移氢解不需在压力条件下进行, 因此反应较温和, 操作简单, 无需耐压设备, 但氢供体 的引入给产物的提纯带来不便, 是该反应的一大缺点.

甲酸作为一种常见有机氢供体, 能与多数有机溶剂 混溶, 是优良的氢供体. 在氨基酸和多肽合成过程中, 催化氢转移氢解被用来脱除芰基保护基, 由于环已烯和 1,4-环已二烯氢供体与肽及肽衍生物不混溶性, 使得在 该条件下的催化氢转移反应不能发生. Elamin 等 ${ }^{[40]}$ 发现 利用低浓度的甲酸溶液如体积分数为 $4.4 \%$ 的甲酸/甲醇 溶液, 在 $\mathrm{Pd} / \mathrm{C}$ 催化下 $10 \mathrm{~h}$ 就能以较高的产率(90\%)脱去 芐基赖氨酸 25 上的 $N$-芐基(Eq. 11).

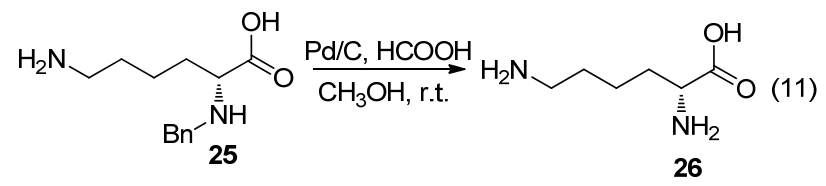

温和的反应条件对那些温度敏感的底物尤为重要, 1987 年 Gray 等 ${ }^{[47]}$ 以甲酸为氢供体对 $N, N$-二苠基丙氨酸 甲酯(27)进行氢解脱苄(Eq. 12), 该反应在室温下氢解 2 $\mathrm{h}$ 即可完成, 氮原子上的两个苄基以较高的产率同时脱 去得到丙氨酸甲酯.

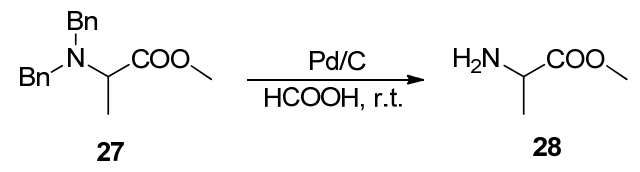

Zheng 等 ${ }^{[48]}$ 在合成 $\beta$-氨基酸 $\mathbf{3 0}$ 时, 以催化氢解进行 氨基脱保护时始终得到脱茮基、脱卤素的混合物. 他们 通过改变溶剂、催化剂、氢供体等条件, 最终发现催化 剂的种类、溶剂对脱茮产物的影响不大，氢供体则影响 脱茮产物的组成. 研究表明, 在 $\mathrm{Pd} / \mathrm{C}-\mathrm{HCOOH}-\mathrm{CH}_{3} \mathrm{OH}$ 体系中，茮基和带有支链保护的茮基均能脱去得到伯 胺, 而且苯环上的卤素并未脱除(Eq. 13). 因此, 可通过 选择适当的氢供体在卤素存在下脱保护.

甲酸铵作氢供体的反应在文献中时有报道，它本身 


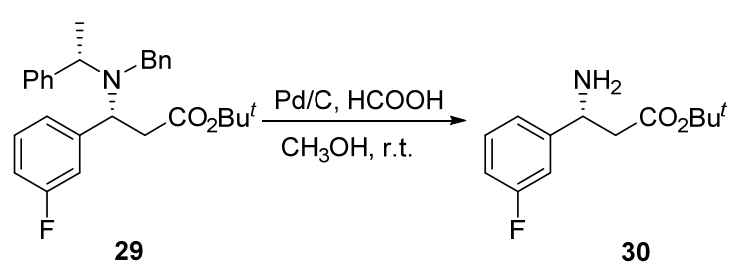

不具酸性且几乎不与底物发生副反应, 在脱保护之后分 离简单. 莫西沙星作为第四代喹诺酮类抗菌药物, 因其 具有半衰期长、不良反应少及抗菌谱广等优点, 具有很 好的应用前景. $(S, S)-2,8$-二氮杂双环 [4.3.0]壬烷作为莫 西沙星的关键中间体, 其快速高效合成是影响莫西沙星 生产及应用的重要因素. Chen 等 ${ }^{[49]}$ 在优化合成 $(S, S)-2,8-$ 二氮杂双环 [4.3.0]壬烷时, 以甲酸铵为氢供体使用催化 转移氢解代替传统的高压催化氢解, 在甲醇溶剂中反应 $4 \mathrm{~h}$ 即可以 $90.5 \%$ 的收率得到 $(S, S)-2,8-$ 二氮杂双环 [4.3.0] 壬烷(32) (Eq. 14). 该方法在常压下就可以进行, 大大降 低了生产成本, 缩短了反应时间, 很好地促进了莫西沙 星在医药领域的应用.

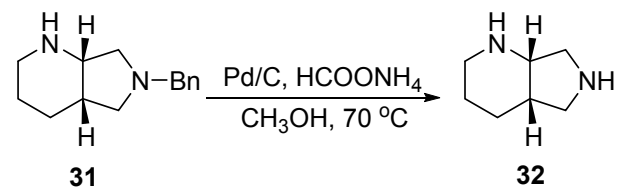

Hedgehog 信号通路可在胚胎阶段调节细胞的增殖 和分化, 其异常激活与肿瘤、癌症的发展有关, 因此通 过 Hedgehog 信号通路发现新的潜在抗癌药物. Taladegib 作为一类已在临床试验的 Hedgehog 信号通路抑制剂, 其高效合成具有重要的研究价值. Guo 等 ${ }^{[00]}$ 改进、优化 Taladegib 合成路线, 使用甲酸铵代替氢气, 用苄基保护 基代替叔丁氧羰基保护基, 发现了一种新奇、高效合成 Taladegib 的新方法. 该方法以甲酸铵为氢源、 $\mathrm{Pd} / \mathrm{C}$ 为催 化剂, $50{ }^{\circ} \mathrm{C}$ 反应 $12 \mathrm{~h}$ 即可高效脱除茮基(Eq. 15). 相比 传统方法，该方法反应条件温和、产率高、后处理简单， 适宜于大规模合成.

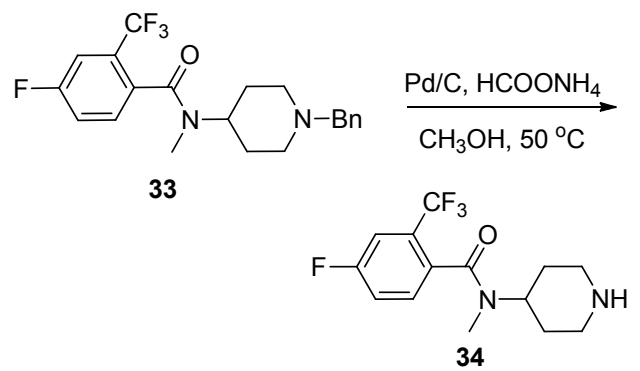

(15)

环己烯和 1,4-环己二烯也可作为催化氢转移的氢供 体. Bajwa 等 ${ }^{[51]}$ 使用 1,4-环己二烯实现了含有 $N$-、 $O$-芐 基底物的选择性脱 $N$-脱苄, 而 $O$-芐基在反应中能不受 影响(Eq. 16).

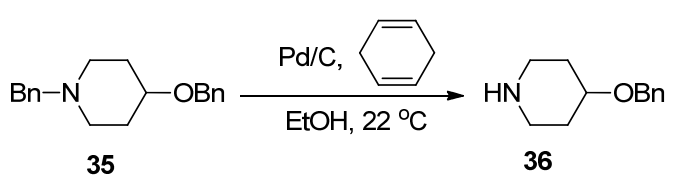

$\mathrm{Babu}$ 等 ${ }^{[52,53]}$ 还报道了一种新型催化转移氢解方法, 即使用廉价的金属镁、锌与氢供体甲酸铵去除 $N-\mathrm{Bn}$ 、 $O-\mathrm{Bn}$ 和 $S-\mathrm{Bn}$ 衍生物的苄基. 在此过程中, 一些取代基 如卤素、甲氧基、苯酚、酯、酸、乙烯基和叔丁氧羰基 (Boc)均不受影响. 该方法使用廉价金属催化很好地克 服了贵金属催化的高成本的缺点，同时避免了自燃，是 一种经济、环保的替代脱苄方案，具有较好的应用前景.

\section{2 氧化脱苄}

还原脱茮虽能脱除大多数有机胺的茮基保护基，但 脱除苄基的同时也容易将其他基团脱除，因此该方法选 择性差. 氧化脱苠是兼具温和和高选择性的脱苠方法, 具体过程为叔胺被氧化剂氧化形成 $\alpha$-羟基苠胺, 随后 $\alpha$ 羟基芐胺进一步分解成仲胺及醛(Scheme 4), 达到脱芐 的目的 ${ }^{[8,9,54]}$. 氧化脱茮所用的氧化剂主要有有 $\mathrm{KMnO}_{4}$ 、 $\mathrm{CrO}_{3} 、 \mathrm{O}_{2}$ 、硝酸铈铵 $(\mathrm{CAN})$ 和 2,3-二氯-5,6-二氰对苯醌 (DDQ)等.

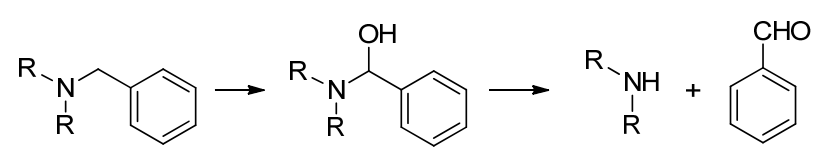

图式 4 氧化脱苄的反应机理

Scheme 4 Reaction mechanism of oxidative debenzylation

CAN 是氧化脱芳中应用最广泛的氧化剂，其具有 反应活性高、条件温和、操作方便、后处理简单、污染 小, 具备一定的选择性等特点. 由于 CAN 在多数有机 溶剂中溶解度低, 在水中溶解度高, 因此 CAN 参与氧 化脱茮反应大多在水、乙腈的混合溶剂中进行 ${ }^{[55]}$.

$N, N$ 二 二苠基是构成 $\mathrm{NH}_{2}$ 基团保护的重要形式，已广 泛应用到亲核加成或周环反应. 然而对于含敏感基团的 底物而言, 催化氢解选择性脱保护得到氨基或单 $N$ - 芐 基化合物的途径是困难的. 在苄基酯或苄基醚存在下含 有两个 $N$-苄基取代基的叔胺 37 , 很容易用 CAN 进行选 择性脱单苄基 ${ }^{[56]}$ (Eq. 17).

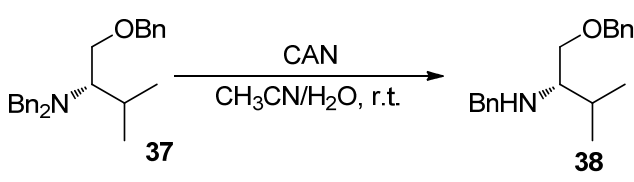

催化氢解是应用最广的脱茮方法, 但是对于含有不 饱和键的底物往往导致双键的还原和脱茮同时发生, 得 
不到期望的脱苄产物. CAN 作为脱茮的氧化剂, 不仅 能高效脱除苄基, 而且对不饱和键显示出一定的化学惰 性, 能够保留底物中的不饱和建. Torres 等 ${ }^{[36]}$ 使用 CAN 氧化苄基保护的氮杂 [4.3.3]螺桨烷(39), 能够脱去苄基 得到裸露的仲胺, 螺桨烷骨架上的双键并未发生变化 (Eq. 18). 该脱茮方法对于含不饱和键的底物具有良好 的选择性, 解决了还原脱苄不具选择性的问题.
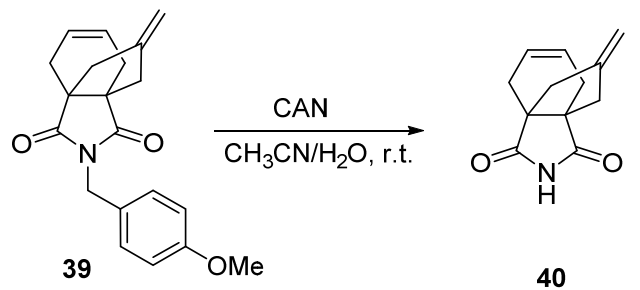

目前, CAN 和 DDQ 已被广泛用作含苄基保护基团 脱保护, 如醇 ${ }^{[57,58]}$ 和胺 ${ }^{[59 ~ 61]}$ 的脱保护. 传统脱茮方法可 以容易地实现杂原子芐基脱保护, 但是仅有少数化合物 在多官能团存在下能实现化学选择性脱芐. Bull 等 ${ }^{[62,63]}$ 以不同底物来进行 CAN 氧化脱芐反应, 详细研究了该 脱芳试剂的反应特点. 研究发现, CAN 和 DDQ 虽然均 能选择性脱除苄基，但两者的脱芐效果存在一定差距， 前者优先脱除 41 中未取代的苄基得到 $\mathbf{4 2}$, 后者则更适 用于对含给电子基团的芐基脱除(Scheme 5). 此外, CAN 对叔胺有较好的脱茮效果, 而对仲胺往往是化学 惰性的. 因此, 在含多芐基的底物中尤其是 $N, N$-二芐基 叔胺 44, CAN 氧化脱茮仅停留在脱单芐的阶段(Scheme 6).

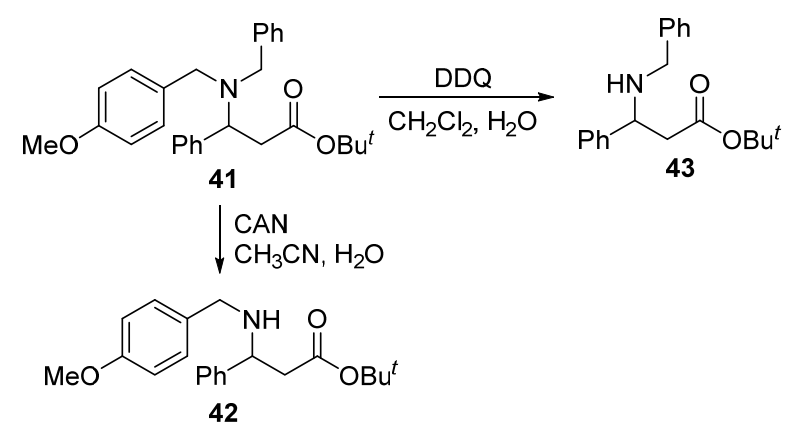

图式 5 两种氧化剂脱苄反应比较活性

Scheme 5 Comparison of the activity of two oxidants for debenzylation<smiles>CC(C)(C)CN(CNCc1ccccc1)Cc1ccccc1</smiles>

图式 6 三苄基胺氧化脱芐

Scheme 6 Oxidative debenzylation of tribenzylamine

陈树森等 ${ }^{[64]}$ 选用多种不同氧化剂对 HBIW 进行了
一系列研究，氧化剂弱，反应不发生; 氧化剂强，笼型 骨架解体. 鉴于 HBIW 脱茮产物含有裸露的 $\mathrm{N}-\mathrm{H}$ 键, 在多数的氧化体系中的稳定性要远低于 HBIW, 因此在 脱茮过程中必须及时保护脱茮产物. 他们采用酸䣶为反 应介质，在冰盐浴中用 $\mathrm{KMnO}_{4}$ 氧化 $\mathrm{HBIW}$ 得到了脱芐 乙酰化的产物 46 (Eq. 19), 而使用 $\mathrm{CrO}_{3}$ 氧化脱茮 ${ }^{[65]}$ 则往 往将茮基氧化成苯甲酰基未得到脱芐的产物.

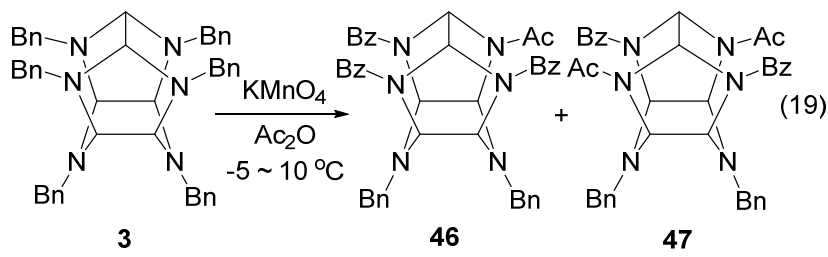

氧气是另一类重要的脱苠反应氧化剂, 尤其适用于 含氮杂环, 在碱性条件下可提高其反应活性. 1983 年, Gigg 等 ${ }^{[66]}$ 首次报道了使用 $t$ - $\mathrm{BuOK}$ 和 $\mathrm{O}_{2}$ 用于吡喃葡萄 糖苷的快速 $N$-脱芳基化的实例, 近 20 年后, Haddach 和 Kong 等 ${ }^{[67,68]}$ 又用相同氧化试剂实现芳香杂环的 $N$-脱苄 基化. Semak 等 ${ }^{[69]}$ 此基础上改进反应条件, 提出了一种 环保，易于处理、经济且高效的脱苄方法，该方法在碱 性介质中使用空气或稀释的氧气作氧化剂, 能快速脱去 取代茮基(Eq. 20). 除此之外, 所需的脱茮基化产物可直 接用于合成而无需进一步纯化，反应后处理仅需要酸碱 中和萃取除去形成的苯甲酸.

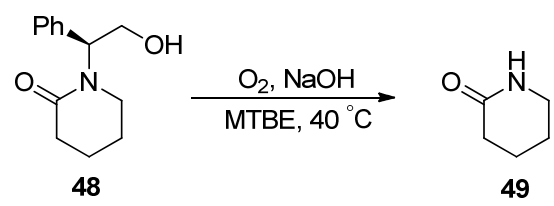

目前, 以 $\mathrm{O}_{2}$ 为氧化剂的脱苠方法经过几十年的发 展, 形成了 $\mathrm{O}_{2}$ 和 $t$ - $\mathrm{BuOK}$ 的氧化脱茮体系. 2015 年, 朱 文通等 ${ }^{[70]}$ 分别以 $N$-取代茮基咔唑和 $N$-取代吲哚为原料, 在碱性条件氧气环境下脱去茮基得到咔唑和吲哚 $\mathbf{5 1}$ (Eq. 21). 该方法操作简单、反应温和且安全环保, 在天 然产物和药物的合成中有重要应用.

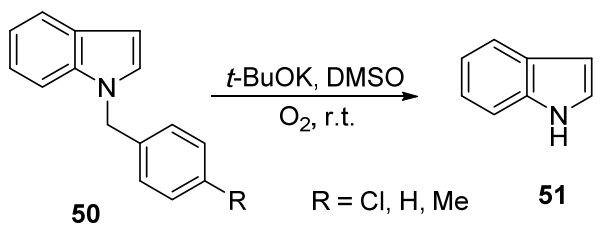

2016 年, Page 等 ${ }^{[71]}$ 发现了一种 1,4-二取代三坐通过 烷基化脱茮基制备 1,5-二取代三唑的方法，该方法未使 用贵金属催化剂，简便高效廉价. 他们首先用碘代烷对 1,4-二取代三唑进行烷基化，生成一种反应活性较高的 
三唑盐 53 , 然后在 $t$ - $\mathrm{BuOK}$ 的存在下氧化脱去苄基 (Scheme 7). 研究同时表明, 碘和 $t$-BuOK 的存在对成功 脱茮有重要作用。

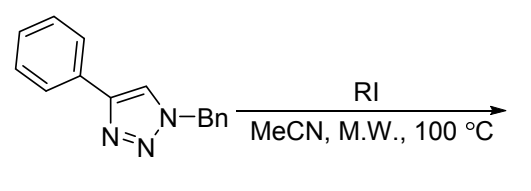

52
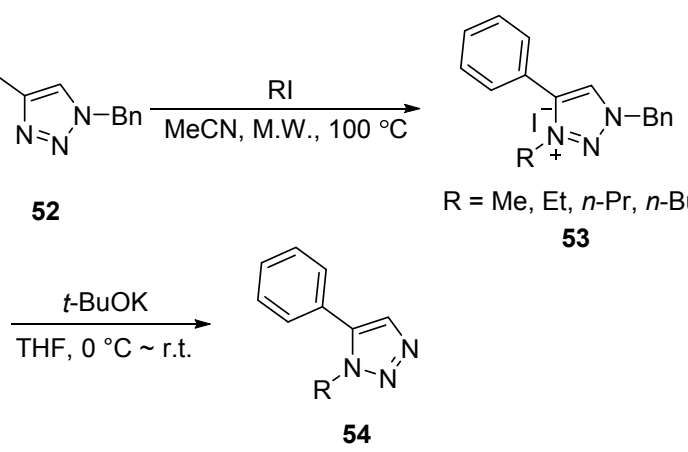

$\mathrm{R}=\mathrm{Me}, \mathrm{Et}, n-\mathrm{Pr}, n-\mathrm{Bu}$

53

图式 7 氧化脱苠合成 1,5-二取代三唑衍生物

Scheme 7 Oxidative debenzylation synthesis of 1,5-disubstituted triazole derivatives

Zhao 等 ${ }^{[72]}$ 在没有配体情况下使用 $\mathrm{Pd}(\mathrm{OAc})_{2}$ 催化剂 催化二取代三唑芳基化偶联得到三取代三唑 56, 反应 混合物不经过分离直接加入强碱 $t$ - $\mathrm{BuOK}$ 实现氧化脱苄 (Scheme 8). 该反应在微波条件短时间内实现偶联、脱 苄一锅转化, 脱苄的底物不需分离提纯, 具有操作简 单、产率高(产率 $60 \% \sim 90 \%$ )、反应条件温和等优点. 微 波的使用降低了反应的难度, 大大加快了反应的进行, 提高了产率.

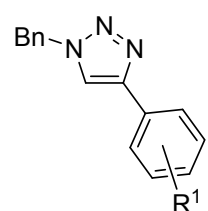

55

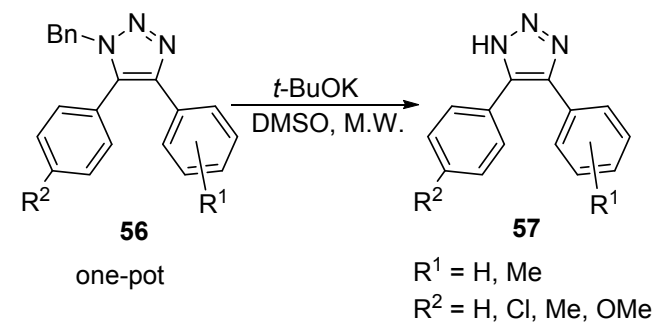

图式 8 氧化脱苄合成二取代三唑

Scheme 8 Oxidative debenzylation synthesis of disubstituted triazole

Feula 等 ${ }^{[73]}$ 将含有叔和仲茮胺官能团的化合物暴露 于碘单质和碳酸铯的氧化条件下, 水解后得到仲胺的脱 茮产物, 证实了仲茮基胺通过氧化、水解是脱茮的有效 方法. 该方法首先用碘单质和碳酸铯作为混合氧化剂在 乙腈中氧化得到亚胺, 随后亚胺经水解得到胺 59 (Eq. 22). 该方案操作简单, 产率高产且无需分离纯化, 能选
择性氧化脱去仲苠基胺上的芐基，而对于叔胺芐基具有 较好的化学惰性. 该方案不仅能有效地脱去茮基, 还可 脱去烷基，这为茮基、烷基的脱保护策略提供了选择.

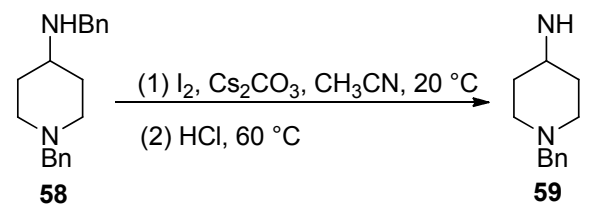

偶氮二甲酸二异丙酯(DIAD)也是一种氧化脱芐的 试剂, 它不仅能脱去 $N$-芐基, 而且在多种官能团存在下 具有选择性. Kroutil 等 ${ }^{[74,75]}$ 提出了选择性脱 $N$-芐基的新 方法，此类试剂尤其适用于多官能团的杂环化合物. 该 方法通过茮基保护的底物与 DIAD 反应可选择性脱除 $N$-芐基(Eq. 23)，而其上的 $O$-芐基并未反应.

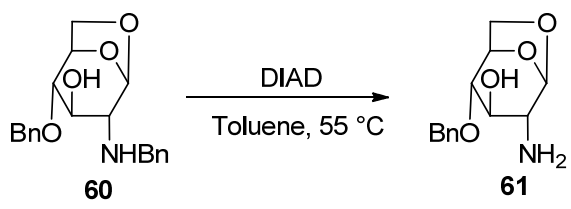

Moriyama 等 ${ }^{[76]}$ 开发了一种由碱金属溴化物/过硫酸 氢钾复合盐(Oxone)氧化体系脱除 $N$-芐基酰胺和 $O$-芐基 醚上茮基的脱苠方法(Eq. 24). 该脱芐方法具有很好的 底物普适性，无论是对氮原子上连有吸电子基团如苯磺 酰基、硝基苯磺酰基、苯甲酰基，还是含有甲基、乙基、 异丙基、异丁基、环戊基、环己基、环庚基等供电子的 烷基，均有很好的脱茮效果. 除此之外，该体系还能脱 去 $O$-苄基醚上苄基得到酮类化合物. 该试剂具有对空 气稳定、易处理、低毒性且不产生污染环境的产物等特 点, 适宜在有机合成中广泛使用.

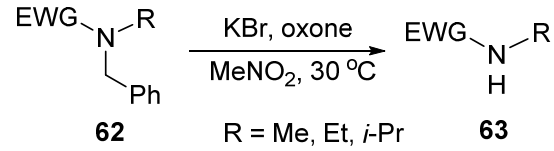

\section{3 强碱条件下脱苄}

传统催化氢解和催化氢转移氢解虽能高效脱去氮 原子上的苄基，但对某些底物如 $N$-酰基- $N$-茮基衍生物 或芳香杂环的脱苄往往不成功. 可溶性碱金属和有机金 属试剂可用于切断 $N$-茮基、 $O$-茮基中 $\mathrm{C}-\mathrm{N} 、 \mathrm{C}-\mathrm{O}$ 键, 是催化氢解的替代方法. 碱金属脱苠的过程是金属的电 子转移到反应底物生成负离子自由基，负离子自由基裂 解产生苄基自由基和胺负离子, 苄基自由基获取氢原子 生成甲苯, 胺负离子获取质子得到胺 ${ }^{[2,9]}$. 近年来, 越来 越多的研究表明，强碱金属和有机金属试剂可用于较难

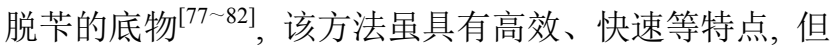
由于该方法中涉及到活泼金属试剂，低温、无氧的操作 
环境，其苛刻的反应条件大大限制了应用.

有机锂化合物是一类重要的金属有机化合物, 独特 的电子分布决定了其强亲核性和强碱性. 因此, 有机锂 具有对多种官能团进行烷基化、脱除保护基团、合成其 它甲基化有机金属试剂、还原其他过渡金属等应用. Suzuki 等 ${ }^{[83]}$ 用甲基锂与苄基取代的吲哚类衍生物 64 反 应, 制得脱苄产物吲哚类似物(Eq. 25). 此外, 正丁基锂 和二异丙基氨基锂(LDA)等有机锂化合物同样用来作为 脱茮基试剂, 且无需在低温下进行，上述试剂的反应条 件较碱金属脱茮温和. 因此, 有机锂化合物广泛地用来 合成吲哚和吲哚类衍生物.<smiles>Cn1c2ccccc2c2ccccc21</smiles>

64

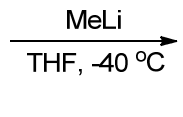

(25)

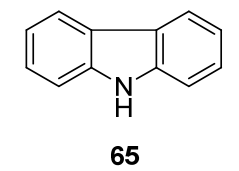

Rao 等 ${ }^{[84]}$ 在合成 atorvastatin 过程中, 以催化氢解、 催化氢转移、叔丁醇钾氧化体系等不同方法均不能成功 脱茮. 最终, 他们发现了将金属钠溶解在液态氨中, 并 以 $t-\mathrm{BuOH}$ 为质子供体, 在 $-78{ }^{\circ} \mathrm{C}$ 低温下能脱去芳香杂 环 66 上的氮苄基(Eq. 26). 该方法虽反应条件苛刻, 并 涉及危险试剂和药品, 但对于传统方法较难脱除的 $N$ 酰基- $N$-苄基衍生物和芳香杂环化合物来说不失为一种 较好的脱苄方法.

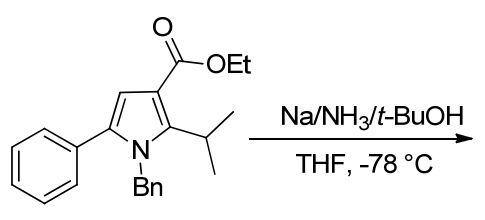

66

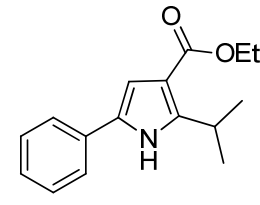

67

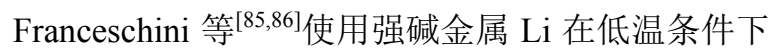
对茮基硫吗啉-3-酮脱保护得到硫吗啉-3-酮 69 (Eq. 27), 随之探索了反应时间对脱苠产物收率的影响. 结果表 明, 随着反应时间的延长, 产物的副产物变多, 致使分 离提纯难度加大, 因此合理控制反应时间是控制产物分 布的有效方法. 该方法虽反应迅速，产率高，且对于茮 基上含有支链的底物也有较好的脱茮效果, 但其反应条 件的苛刻及试剂的危险性严重限制了该方法的实用性.

负载于多孔硅胶(SG)的碱金属是有机胺还原脱烯 丙基、茮基、三苯甲基化的有效试剂(Eq. 28) ${ }^{[87]}$, 这类试 剂为传统碱金属氨溶液脱保护提供了方便替代品. 其 中, 乙二胺(EDA) 是促进脂族底物反应所必需的添加物, 随后底物可在 16 24 h 内将其彻底脱保护. 该方法具有 反应简单, 易于后处理和分离的特点, 反应条件也较碱 金属氨溶液脱茮温和。

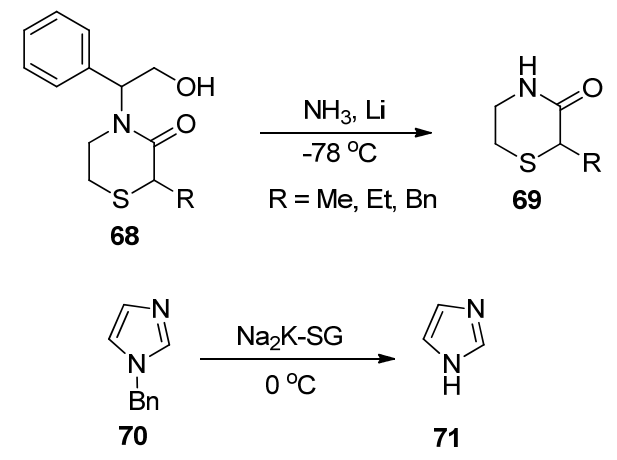

$\mathrm{LiAlH}_{4}$ 作为强还原剂在有机合成中占据重要的地 位，尤其是用于酯、羧酸和酰胺的还原. Page 等 ${ }^{[71]}$ 发现 了一种由 1,4-二取代三唑 72 通过烷基化而后脱苠基制 备 1,5-二取代三唑的方法(Scheme 9), 该方法未使用贵 金属催化剂，简便高效廉价. 他们首先用碘代烷对 1,4二取代三唑进行烷基化，生成一种反应活性较高的三唑 盐. 由于三唑盐中含有不饱和的五元杂环，无法通过使 用催化氢解、氢转移氢解进行脱苄. 他们尝试使用强还 原试剂 $\mathrm{LiAlH}_{4}$ 还原三唑盐, 意外地发现能够脱茮得到 1,5-二取代三唑. $\mathrm{LiAlH}_{4}$ 进行脱茮虽获得目标物产率较 低，但开拓了一种脱去杂环上茮基的新方法.

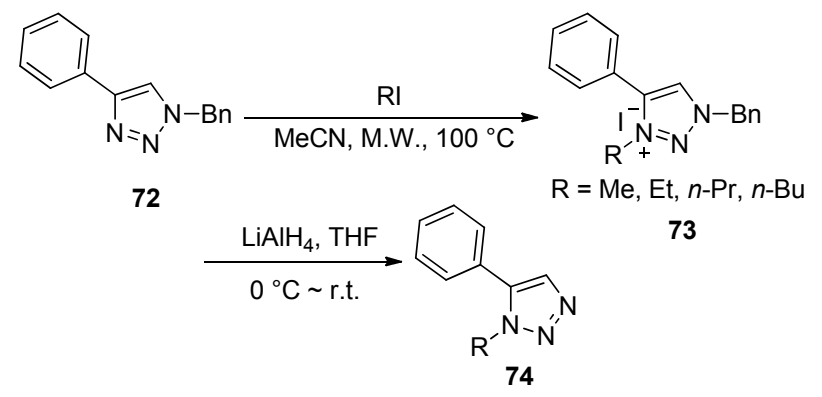

图式 $9 \mathrm{LiAlH}_{4}$ 为脱茮试剂的强碱脱茮

Scheme 9 Strong alkali debenzylation of $\mathrm{LiAlH}_{4}$ as debenzylation reagent

截短侧耳素(pleuromutilin)是一类天然存在的抗生 素, 具有一定的抗菌活性, 其中截短侧耳素 SB-268091 已被开发用来治疗鼻窦炎和中耳炎. 2012 年, Choudary 等 ${ }^{[88]}$ 报道了抗生素 SB-268091 的合成, 其中重点介绍了 合成奎宁环-4-硫醇配体 76 新途径. 该途径涉及到在液 氨中使用金属钠进行还原性脱茮基，实现了 $N$-茮基和 $S$-苄基同时脱除(Eq. 29). 钠与液氨组成的脱苄体系具 有较强的脱苄能力, 因此对脱保护不具备选择性, 特别 适用于不含敏感基团且较难脱除的茮基化合物的脱保 护.

\section{4 酸解脱苄}

与其他脱苄方法相比, 酸解脱苄反应迅速且操作简 


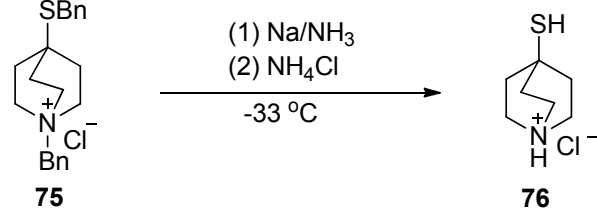

单, 因此也是一类重要的脱芐方法. 酸解脱芐就是芐基 保护的底物在酸的作用下, 脱除苄基得到游离胺或有机 胺盐的过程 ${ }^{[4]}$. 酸解脱芐过程需要在酸性环境中进行, 因此底物需对酸具有较好的耐受力, 常用催化酸解脱苄 的酸有三氟乙酸(TFA)、三氟甲磺酸(TfOH)、对甲苯磺 酸 $(p-\mathrm{TsOH})$ 等. 酸解脱芐过程操作简单, 反应迅速, 但 是仅对某些底物有较好的脱苄效果, 不具备通用性.

吡唑是一类具有广泛药物活性的基团, 近年来大量 吡唑衍生物被合成出来. 尤其值得注意是含有 1-芳基吡 唑结构的 celecoxib (78), 它是选择性环氧酶-2 (COX-2) 抑制剂，可用于治疗骨关节炎、类风湿性关节炎和急性 疼痛等症状. 2012 年, Shaw 等 ${ }^{[89}$ 报道了一种通过 Ugi 反 应/脱苄基化/肼介导的环化的三步反应来合成 3-差基吡 唑的方法. 该方法脱苄基时, 采用 10\% TFA(三氟乙 酸) $\mathrm{DCE}$ (二氯乙烷)溶液在微波辅助 $10 \mathrm{~min}$ 下脱去苄基 保护的 3-羟基吡唑衍生物的苄基，产率也超过 80\% (Eq. 30). 相比于其他传统脱芐方式，该方法快速高效，且脱 苄产物的分离也较简单.<smiles></smiles><smiles>O=C(Nc1c(O)n[nH]c1-c1ccccc1)c1ccc(Br)cc1</smiles>

Lee 等 ${ }^{[90]}$ 在制备 $N$-甲基酰胺的过程中，首先合成出 $N$-甲基- $N$-芐基酰胺，随后经酸解脱芐的方法合成了 $N$ 甲基酰胺. $N$-甲基- $N$-苄基酰胺 79 以三氟乙酸(TFA)为脱 苄试剂得到 $N$-甲基酰胺(Eq. 31), 而酰胺和芳胺上的 $N$ 甲基均能保持稳定. 该方法对于合成各种含氮酰胺非常 有效, 并且应用于多种在酸性下稳定的官能团的脱保 护.

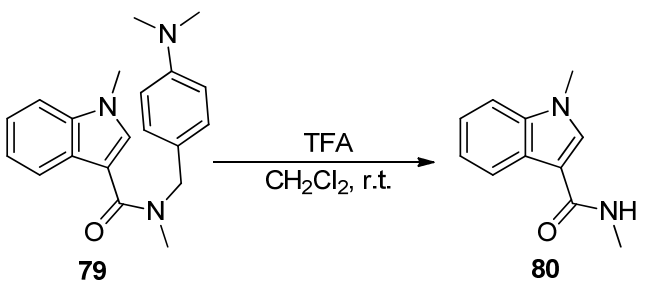

三氟乙酸不仅能脱去苄基，对于取代苄基如对甲氧
基苄基也有不错的脱苄效果. 吲哚环是许多天然产物和 药物的主要骨架，具有一定替代模式的吲哚的高效构建 具有挑战. 2014 年, Lam 等 ${ }^{[91]}$ 在研究高效构建吲哚环时, 需对重要中间体 5-丁基-2-碘-3-[ $N$-(4-甲氧基苠基)- $N$-(对 甲苯磺酰)氨基]苯基甲磺酸(82)脱保护。他们使用三氟 乙酸为脱茮试剂, 在室温环境中搅拌 $3 \mathrm{~h}$ 即可脱去对甲 氧基苠基(Eq. 32)，产率达 94\%～99\%。该方法既不用使 用耐压的设备, 又不需要昂贵的催化剂, 在温和的条件 下就可实现脱茮，极大降低了合成吲哚的成本.

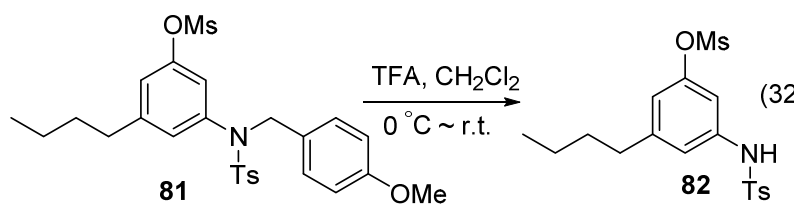

另外, Rombouts 等 ${ }^{[22]}$ 报道了在微波辅助下用三氟 甲磺酸对 $N$-芐基酰胺 83 进行脱苄基的新方法(Eq. 33). 该脱芳方法已经显示出相当普遍的适应性，对于仲、叔， 脂族、芳香族和非环状、环状酰胺均有较好的脱茮活性.<smiles>[R]c1c(-c2cc(C)no2)c(=O)n(Cc2ccccc2)c2ccccc12</smiles>

2014 年, Lima 等 ${ }^{[93]}$ 报道了 kealiiquinone 及 Leucetta 衍生物的合成方法(Eq. 34). 在由苄基保护基的咪唑萗 醌合成 6,7-二甲氧基-1-甲基-4-苯基- $1 H$-菜并 [2,3- $d$ ]-味 唑-2,5,8-三酮(86)时, 由于咪唑菜醌化合物中含有羰基、 甲氧基和 $N$-甲基, 传统的还原脱茮和氧化脱茮的脱苄 方法均不能成功脱保护. 随后他们改用 $\mathrm{TfOH}$ 作为酸解 脱茮的试剂, 在 $55{ }^{\circ} \mathrm{C}$ 即可脱去苄基得到 86, 而骨架上 的羰基、甲氧基及 $N$-甲基也并未发生变化.

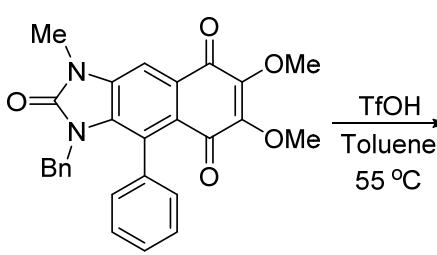

85<smiles>COC1=C(OC)C(=O)c2c(cc3c([nH]c(=O)n3C)c2-c2ccccc2)C1=O</smiles>

Chern 等 ${ }^{[94]}$ 发现了用对甲苯磺酸脱去苄基的新方 法，该方法尤其适用于对酸或还原体系敏感的底物. Chern 等使用同类底物 87 对不同的脱茮方法进行比较, 以 $\mathrm{Pd} / \mathrm{C}$ 为催化剂的催化氢解和三氟乙酸的酸解脱茮的 产率均在 $22 \%$ 以下, 而对甲苯磺酸的酸解脱苄却能在 $90 \%$ 以上. 此外, 对甲苯磺酸还能在 $N$-Fmoc、 $N$-Boc 或 $N$ - Tr 保护基存在下, 选择性脱除苠基，与传统催化氢解 
相比，该方法都具有独特的优势(Eq. 35).

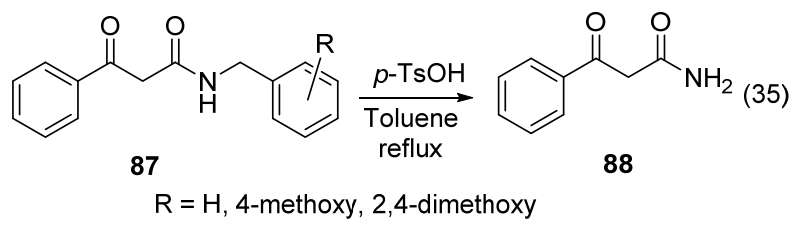

\section{5 其它方法脱苄}

\section{1 硝解脱苄}

硝解脱苄即底物中的茮基与硝化试剂反应得到硝 基或亚硝基取代的衍生物，该方法实现了含能材料的脱 芐和硝化的一步转化, 在含能材料的合成中具有重要应 用. 硝解脱茮就是硝化试剂首先进攻氮原子, 随后脱去 差胺得到亚胺正离子, 亚胺正离子经水解硝化得到硝基 或亚硝基取代的衍生物的过程 ${ }^{[8,9]}$. 由于反应常在酸性 条件下进行且需使用硝化试剂, 因此底物需具有较好的 稳定性, 这大大限制了它的使用. 即便如此, 这仍是直 接引入硝基的脱茮方法, 在含能领域有着独特的优势.

亚硝解 ${ }^{[95,96]}$ 也可用于芐基的脱保护，即分子中芐基 保护基被亚硝基取代. 由于亚硝基易被氧化成硝基, 因 此亚硝解脱茮在含能领域也有重要应用. 于永忠等以四 乙酰基二苄基异伍兹烷(4)为原料, 在 $\mathrm{NOBF}_{4}$ 体系中亚 硝解得到了亚硝解的脱芐产物. 该方法可将催化氢解不 易脱除的 4,10位的芐基脱掉, 获得四乙酰基二亚硝基异 伍兹烷(Eq. 36), 后者经进一步氧化、硝化可得到 HNIW. 此后, Bayat 等 ${ }^{[97 ~ 99]}$ 又改用 $\mathrm{N}_{2} \mathrm{O}_{4}$ 亚硝化试剂将 4,10 位 不易脱除的芐基脱除, 得到四乙酰基二亚硝基异伍兹 烷.

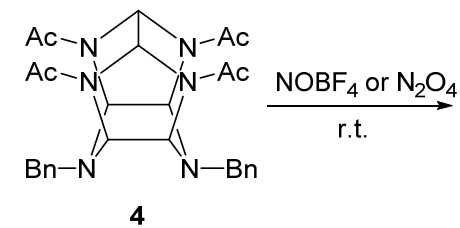

4

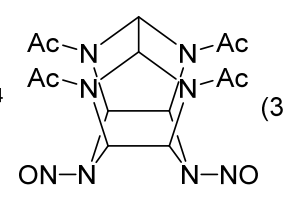

89

\section{2 取代脱茮}

取代脱芳就是一种以易脱除的基团取代底物中的 苄基得到取代衍生物, 而后将易取代的基团脱去得到裸 露的 $\mathrm{N}-\mathrm{H}$ 键的方法. 取代脱茮的取代基一般优先选择 氯甲酸酯, 用来进行脱芐的氯甲酸酯有氯甲酸甲酯、氯 甲酸乙酯和氯甲酸苯酯, 其中苯酯的脱芐效率要高于甲 酯和乙酯 ${ }^{[100]}$. 苄基保护的叔胺与氯甲酸酯生成氨基甲 酸酯, 随后氨基甲酸酯在一定条件下反应得到仲胺.

1993 年 Yang 等 ${ }^{[101]}$ 利用氯甲酸氯乙酯取代叔胺 90 上的芐基, 而后经 $\mathrm{CH}_{3} \mathrm{OH}$ 回流得到仲胺的盐酸盐
(Scheme 10). 在反应中, 氯甲酸氯乙酯与叔胺形成季铵 盐, 随后分子内脱去一分子氯茮得到化合物 91, 化合物 91 与甲醇加成脱羧后制得仲胺盐酸盐 92 . 氯甲酸酯脱 茮也是一类重要的脱保护方法, 它适用于多种含氮五元 及六元杂环叔胺, 氯甲酸酯的毒性限制了该方法的使 用.

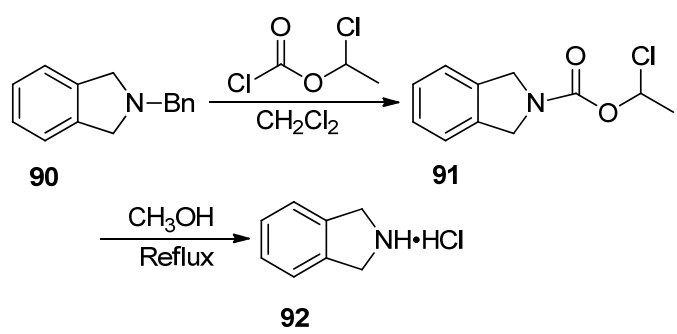

图式 10 氯甲酸氯乙酯为脱茮试剂脱䒾

Scheme 10 Debenzylation of chloroethyl chloroformate as debenzylation reagent

1994 年, See 等 ${ }^{[102]}$ 报道了将 HBIW 与氯甲酸三甲基 硅基乙酯在四氢呋喃或乙醚中进行取代脱芐反应，可制 得六取代的六氮杂异伍兹烷 93 (Eq. 37). 该方法所需温 度低，时间长，并且涉及到有毒的光气，因此实用价值 较小。

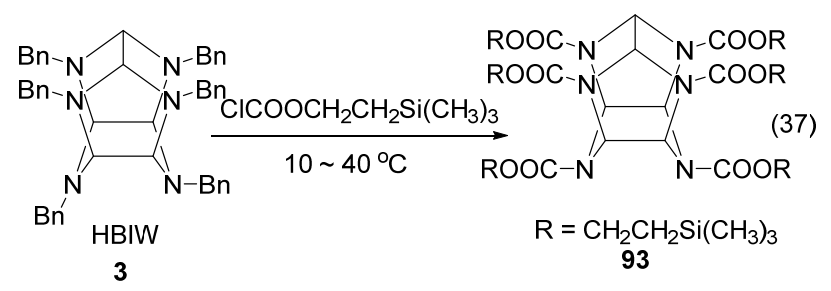

Wright 等 ${ }^{[103]}$ 也利用氯甲酸乙酯作脱苄试剂, 在以 苯为溶剂回流条件下对氮茮基氨基甲酸酯化合物 94 进 行脱苄得到氨基甲酸酯衍生物(Eq. 38). 该方法尤其适 用于制备氨基甲酸酯衍生物，同时发现苯环上含有吸电 子基团或是供电子基团对于脱苄均反应是不利的.<smiles>CCOC(=O)OCCOC(=O)OCCN(C)CCN(C(=O)OCC)c1ccccc1</smiles>

Olofson 等 ${ }^{[104]}$ 发现氯甲酸氯乙酯与叔胺生成季铵 盐，该季铵盐可在甲醇的回流条件下脱去烷基得到胺的 盐酸盐 ${ }^{[103]}$. 因此, 可利用该方法高效脱除氮原子上的 烷基, 并生成季铵盐. 对于脱保护之后不稳定的化合物, 季铵盐的生成可可大大提高产物的稳定性, 在不稳定化 合物的合成中具有独特的优势. 鉴于烷基保护基的脱除 难度大于茮基的脱除，因此该方法也可用来脱除茮基. 
Kanth 等 ${ }^{[105]}$ 使用氯甲酸氯乙酯取代了三取代吡咯衍生 物 96 上的苄基生成氨基甲酸酯衍生物, 在 $\mathrm{I}_{3} \mathrm{~B} / \mathrm{N}\left(\mathrm{C}_{2} \mathrm{H}_{5}\right) \mathrm{Ph}$ 存在下以 $80 \%$ 总产率生成二取代吡咯衍 生物 98 (Scheme 11).
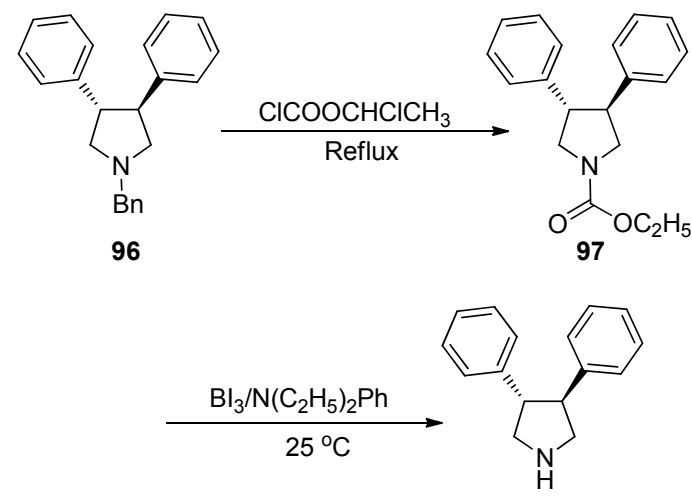

98

图式 11 氯甲酸氯乙酯为脱苄试剂合成二苯基吡咯 Scheme 11 Synthesis of diphenylpyrrole by chloroethyl chloroformate as debenzylation reagent

TeocCl [Teoc: $\mathrm{C}(\mathrm{C}=\mathrm{O}) \mathrm{O}\left(\mathrm{CH}_{2}\right)_{2} \mathrm{TMS}$ ] 也可作为脱苄 试剂, 它也能高效取代苄基生成 $N$-Teoc 衍生物, 随后 $N$-Teoc 衍生物通过 CsF 在 $90{ }^{\circ} \mathrm{C}$ 下即可脱去 Teoc 保护 基, 得到四氢异喹啉 101. Igarashi 等 ${ }^{[06]}$ 合成奎宁类生物 碱前体时, 使用 Teoc 为保护基取代茮基并用 $\mathrm{CsF}$ 脱除实 现了高效构建奎宁环(Scheme 12).

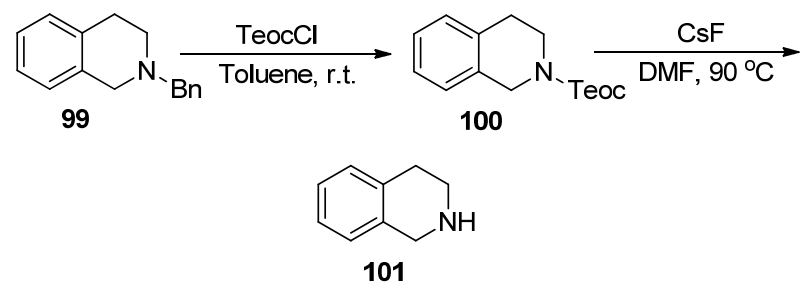

图式 $12 \mathrm{CsF}$ 为脱苄试剂脱苄

Scheme $12 \mathrm{CsF}$ as debenzylation reagent for debenzylation

天然产物(NPs) 是生物活性小分子的重要来源, 其 为发现新药物提供了可能. 在过去 30 年批准的新药中, 大约三分之一的小分子药物是基于天然产物及其衍生 物. NPs 除了具有复杂的结构和化学多样性外, 还因其 惊人的药物分子特性而获得大量关注. Annamalai 等 ${ }^{[107]}$ 合成了六类具有一定生物活性化合物, 试图由此建立一 个具有生物活性的核心结构的化合物库. 他们在合成十 氢-1,6-二氮杂芸类化合物 103 过程中, 由于氮杂䒬上含 有酯, 使用传统的催化氢解不能使苄基保护的氮杂䒺脱 保护. 经过大量的实验, 他们使用 1-氯甲酸氯乙酯成功 脱去 $N$-芐基(Eq. 39), 脱茮产率最高可超过 90\%.

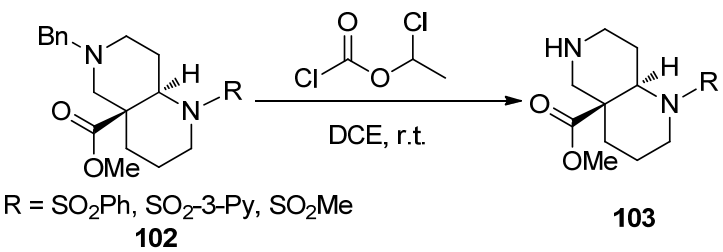

\section{3 单电子转移脱茮}

Pandey 和 Rani 等 ${ }^{[108]}$ 发现了单电子转移脱茮的新方 法, 即以 9,10-二氰基葱作为电子受体, 底物在光照条件 下由基态络合物发生单电子转移, 同时自身被氧气及碳 亲核试剂进攻后得到亚胺正离子, 亚胺正离子再进行水 解得到脱苄的产物 107 和苯甲醛(Scheme 13), 反应的产 率在 75\% 90\%之间. 该方法适应多种底物如环叔胺和 脂肪叔胺, 同时羟基及酯基能在该反应中保持稳定.

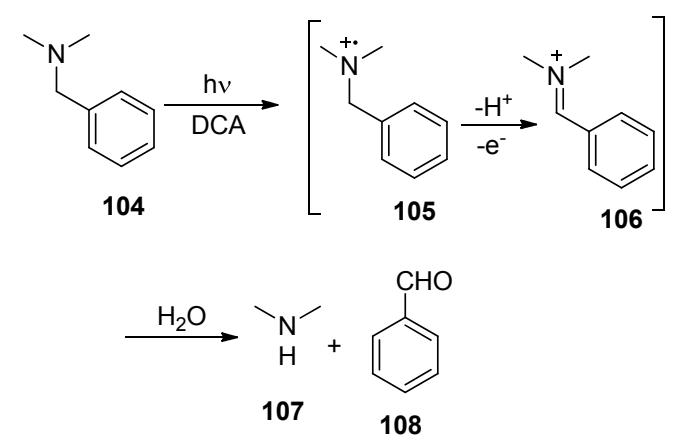

图式 13 单电子转移脱茮

Scheme 13 Single electron transfer debenzylation

叔丁醇钾-三乙基硅烷体系不仅能够实现芳烃和杂 环芳烃的硅烷基化，而且还能还原性断裂 $\mathrm{C}-\mathrm{N}, \mathrm{C}-\mathrm{O}$ 键和芳香硫梄的 $\mathrm{C}-\mathrm{S}$ 键. Smith 等 ${ }^{[109]}$ 系统研究了叔丁 醇钾-三乙基硅烷体系脱保护的反应机理, 包含了单电 子转移和氢化物传递的过程. $\mathrm{Et}_{3} \mathrm{SiH}$ 与 $t$ - $\mathrm{BuOK}$ 首先形 成良好的电子供体，电子经过转移形成中间体 110 , 最 后通过氢化物的传递得到脱茮产物 111 (Scheme 14)以 及副产物甲苯. 该体系由于具有较强的供电子能力, 还 可作为电子供体还原不饱和键双键和参键, 更能脱去甲 基、烯丙基、苄基、芸基等保护基.

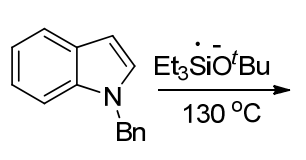

109

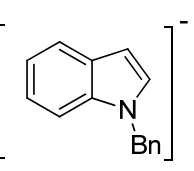

110

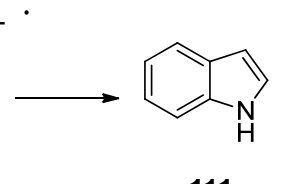

111
图式 14 叔丁醇钾和三乙基硅烷为脱芐试剂的脱芐 Scheme 14 Debenzylation of potassium tert-butoxide and triethylsilane as debenzylation reagent

\section{4 格氏试剂脱苄}

格氏试剂具有强亲核性, 在有机反应中具有重要应 
用, 是增加碳链长度和进行衍生化的重要试剂. Golantsov 等 ${ }^{[110]}$ 在使用 2 -氰基吲哚(112)合成含有手性取代 基的吲哚衍生物过程中, 发现该反应得到的并非是单一 的吲哚酩, 而是含有手性取代基的吲哚酮和脱去苄基的 吲哚酮的混合物(Eq. 40), 从而得到了意外的脱茮结果. 进一步研究表明, 改变反应条件可控制两者的生成比 例, 在乙醚中往往得到两者的混合物, 而在甲苯中仅得 到脱苄基吲哚酮. 此外过渡金属试剂 $(\mathrm{CuCN})$ 的加入使 产物更多地向含有手性取代基的吲哚酮转化. 该方法虽 底物的适应性较低, 但其完成某些含氮化合物衍生化和 脱芐一步转化, 减少了反应步骤.
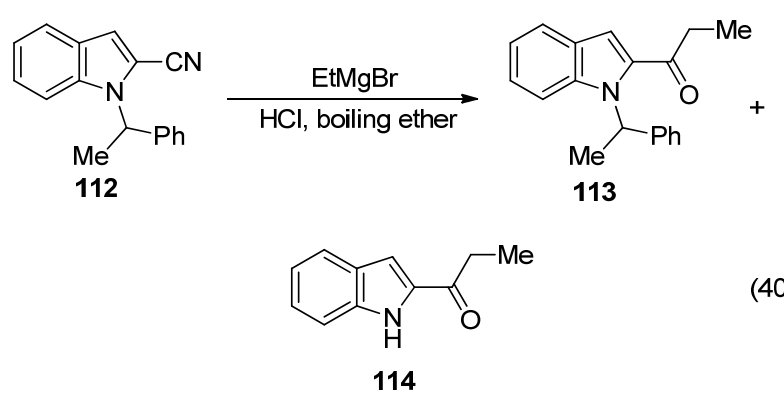

\section{5 酶催化脱苄}

近年来, 因生物催化脱保护不需高温、高压和有毒 的试剂, 对环境影响小, 同时具有高选择性脱保护的特 点, 该方法已成为脱保护的一大热点. 其中, 水解或氧 化还原酶已经被证明可用于多种官能团选择性修饰, 酰 基转移酶、酷酶、脂肪酶和蛋白酶被认为是烷基酯脱保 护的有效酶, 氧化还原酶也已显示出 $N$-脱保护反应的 潜力. Montero 等 ${ }^{[111]}$ 报道了利用漆酶(Laccase)有效脱除 $N$-芐基反应的研究, 漆酶可在水溶液与不同的化学氧化 剂兼容, 这扩大了它们在许多工艺中的适用性. 以漆酶 和 2,2,6,6-四甲基哌啶一氮-氧化物(TEMPO)组成的混合 氧化体系可以实现脂肪胺、环状和芳香胺高选择性温和 脱保护(Eq. 41). 该反应对 $N$-芰基化的仲胺表现出较好 的反应活性, 但它不会改变 $O$-茮基化的醇或 $N$-芐基化 的叔胺, 这是其他化学脱保护方法较难实现的, 适合广 泛用于合成化学, 如氨基酸或核苷化学.

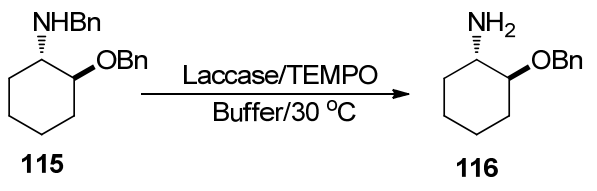

\section{6 其它试剂脱苄}

$N$-碘代琥珀酰胺(NIS)是一种高效、温和、方便且可 控的脱芐试剂, 其能进行 $N$-二芐胺的选择性脱茮基. Grayson 等 ${ }^{[112]}$ 在进行 $N$-卤化生成亚胺的研究过程中, 发
现连续卤化、消除和水解可能为 $N$-脱芐基化提供途径. 他们进一步研究证实, $N$-氯代丁二酰亚胺脱苄效果不佳, 但 $N$-碘代丁二酰亚胺(NIS)能较好实现二芐胺的脱苄基 化 (Scheme 15). 当底物 118 与 NIS 的物质的量比为 $1: 3$ 时得到的是脱单苄产物, 而物质的量比增加至 $1: 6$ 时 则得到较多的脱二芐产物. 该试剂在多种底物中具有实 用性和选择性，尤其是在合成含多官能团苠氨基醇类化 合物，可以通过调节 NIS 的用量来控制反应产物.

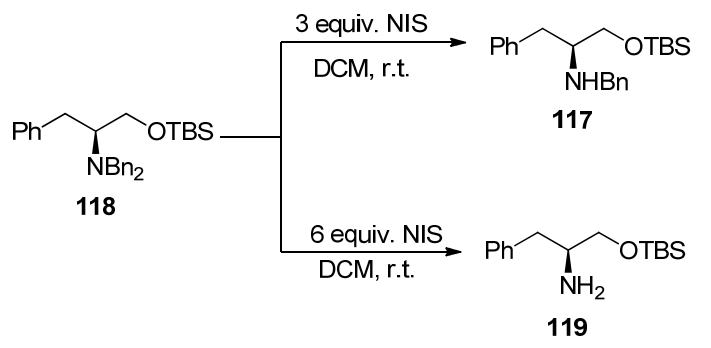

图式 15 NIS 为脱茮试剂脱茮

Scheme 15 NIS as debenzylation reagent for debenzylation

Baker 等 ${ }^{[113]}$ 报道了一种使用 $N$-溴代丁二酰亚胺 (NBS)/偶氮二异丁腈(AIBN)试剂脱去酰胺茮基的新方 法，该方法苄基保护的底物与 NBS 发生澳代反应生成 中间体，而后中间体经水解后发生 $\mathrm{C}-\mathrm{N}$ 键的断裂，生 成脱茮产物 122 (Scheme 16). 脱保护的过程中形成的苄 卤中间体水解是脱苄反应的关键，因此该体系仅对氮原 子上的苄基有较好的反应活性，对于 $N$-甲基和 $N$-苯基 均不能反应.

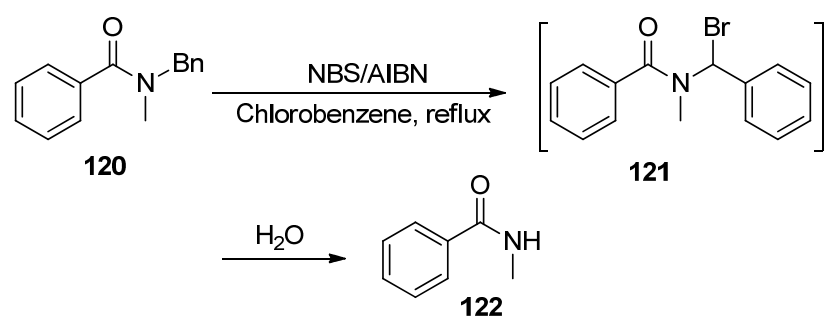

图式 $16 \mathrm{NBS} / \mathrm{AIBN}$ 脱去 $N$-芐基酰胺苄基

Scheme 16 Removal of the benzyl group of $N$-benzyl amide using NBS/AIBN

Yanada 等 ${ }^{[114]}$ 在合成羟吲哚类化合物 124 时, 分别 尝试了以 $\mathrm{BBr}_{3} 、 t-\mathrm{BuOK} / \mathrm{O}_{2} 、 \mathrm{CAN}$ 等氧化脱芐方法, 遗 憾的是以上方法均不能脱去氮原子上的苄基. NBS/AIBN 组成的体系能以 $95 \%$ 的产率脱去茮基得到羟 吲哚(Eq. 42), 该体系为多种天然产物的全合成尤其是 含氮杂环脱保护提供了一种重要的策略.

\section{6 结论与展望}

综上所述, 脱茮的方法有很多, 各有特点. 催化氢 解和催化氢转移氢解脱苄是使用最广泛的方法, 其优点 


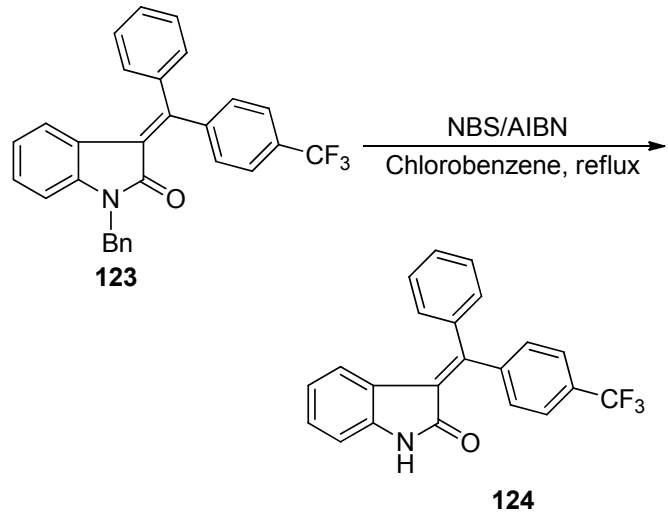

是反应温和, 产率高, 但也存在选择性差、成本高, 往往 不适用于含不饱和键底物的缺点. 催化氢解一般需要在 加压环境中进行, 对反应设备要求高. 催化氢转移氢解 过程中使用的氢供体, 不利于产物的分离提纯. 对于在 反应条件下不稳定的化合物, 可通过预先成盐或是用其 他试剂及时捕捉脱茮产物的方法实现脱保护. 碱金属脱 䒾反应活性高, 产率好, 可用于底物结构稳定、茮基不 易脱除的转化; 但其反应条件苛刻, 涉及有毒危险试剂, 严重制约了其使用范围. 取代脱茮将茮基转换为易脱除 的基团，大大降低了脱茮过程的难度，但不足之处是增 加了转化的步骤. 氧化脱茮虽然操作方便, 后处理简单, 且具备一定的化学选择性, 但在氧化过程中往往会发生 一些副反应, 给脱苄产物的分离、提纯带来了一定的困 难, 同时也对底物的稳定性提出了特殊要求. 硝解脱茮 得到特定的硝基或亚硝基化合物，可直接用来制备含能 材料, 因此在含能材料领域应用较多. 其他脱苄方法虽 然效率高, 但仅针对特定的反应底物, 适应性小. 酶催 化脱芳是目前兼具高效、选择性、环保等特点且前景较 好的脱苄方法, 值得进行深入研究. 但在前期研究中需 进行酶活性篮选，䇻选难度大，强度较高，严重制约了 其应用. 因此, $N$-茮基脱除方法要根据具体反应底物的 结构进行合理选择，选用不同的脱苄策略.

\section{References}

[1] Rong, G.-B. Protective Groups in Organic Synthesis, East China University of Science and Technology Press, Shanghai, 2004, pp. $494 \sim 654$ (in Chinese).

(荣国斌, 有机合成中的保护基, 华东理工大学出版社, 上海, 2004, pp. 494 654.)

[2] Huang, P.-Q.; Jin, L.-R.; Chen, A.-Q. Organic Synthesis, Higher Education Press, Beijing, 2006, pp. 385 389 (in Chinese). (黄培强, 靳立人, 陈安齐, 有机合成, 高等教育出版社, 北京, 2006, pp. 385 389.)

[3] Wu, Y.-L.; Yao, Z.-J.; Hu, T.-S. Modern Organic Synthetic Chemistry, Science Press, Beijing, 2006, pp. $27 \sim 73$ (in Chinese). (吴毓林, 姚祝军, 胡泰山, 现代有机合成化学, 科学出版社, 北 京, 2006, pp. 27 73.)

[4] Wu, Q.-P.; Li, S.-M. Protective Chemistry, Chemical Industry Press, Beijing, 2007, pp. 239 277 (in Chinese). (武钦佩, 李善茂, 保护基化学, 化学工业出版社, 北京, 2007, pp. $239 \sim 277$.

[5] Mackie, R. K.; Smith, D. M.; Aitken, R. A.; Meng, G. Guidebook to Organic Synthesis, Chemical Industry Press, Beijing, 2009, pp. $210 \sim 223$ (in Chinese)

(Mackie, R. K.; Smith, D. M.; Aitken, R. A.; Meng, G. 有机合成指 南, 化学工业出版社, 北京, 2009, pp. 210 223.)

[6] Dong, Y.-S.; Dai, S.-A.; Pan, Z.-F.; Chen, J.-L. Ind. Catal. 2011, 19 , 4 (in Chinese). (戴云生，董守安，潘再富，陈家林，工业催化, 2011, 19, 4.)

[7] Bayat, Y.; Ebrahimi, H.; Fotouhi, F. F. Org. Process Res. Dev. 2012, 16, 1733.

[8] Jia, H.-P.; Ou, Y.-X.; Chen, B.-R.; Xu, Y.-J.; Chen, J.-T. Chin. J. Energ. Mater. 1998, 6(4), 145 (in Chinese). (贾会平，欧育湘，陈博仁，徐永江，陈江涛，含能材料，1998, 6(4), 145.)

[9] Qiu, W.-G.; Yu, Y.-Z. Chin. J. Synth. Chem. 1998, 6, 34 (in Chinese). (邱文革, 于永忠, 合成化学, 1998, 6, 34.)

[10] Davies, S. G.; Ichihara, O. Tetrahedron Lett. 1999, 40, 9313.

[11] Couturier, M.; Andresen, B. M.; Jorgensen, J. B.; Tucker, J. L.; Busch, F. R.; Brenek, S. J.; Dube, P.; Ende, D. J.; Negri, J. T. P. Org. Process Res. Dev. 2002, 6, 42 .

[12] Amat, M.; Llor, N.; Hidalgo, J.; Escolano, C.; Bosch, J. J. Org. Chem. 2003, 68, 1919 .

[13] Davies, S. G.; Nicholson, R. L.; Price, P. D.; Roberts, P. M.; Rueesell, E. D.; Savory, E. D.; Smith, A. D.; Thomson, J. E. Synlett 2004, 901.

[14] Srimurugan, S.; Murugan, K.; Chen, C. Chem. Pharm. Bull. 2009, $57,1421$.

[15] Davies, S. G.; Fletcher, A. M.; Hughes, D. G.; Lee, J. A.; Price, P. D.; Roberts, P. M.; Russell, A. J.; Smith, A. D.; Thomson, J. E.; Williams, O. M. H. Tetrahedron 2011, 67, 9975.

[16] Leitch, D. C.; Greene, T. F.; O'Keeffe, R.; Lovelace, T. C.; Powers, J. D.; Searle, A. D. Org. Process Res. Dev. 2017, 21, 1806.

[17] Yoshida, K.; Nakajima, S.; Wakamatsu, T.; Ban, Y. Heterocycles 1988, 27, 1167.

[18] Gong, X.-B.; Sun, C.-H.; Pang, S.-P.; Zhang, J.; Li, Y.-C.; Zhao, X.-Q. Chin. J. Org. Chem. 2012, 32, 486 (in Chinese). (公绪滨，孙成辉，庞思平，张静，李玉川，赵信岐，有机化学 2012, 32, 486.)

[19] Bellamy A. J. Tetrahedron 1995, 51, 4711.

[20] Wardle, R. B.; Edwards, W. W. WO 97/20785, 1997.

[21] Koskin, A. P.; Simakova, I. L.; Parmon, V. N. Russ. Chem. Bull. 2007, 56, 2370.

[22] Kalashnikov, A. I.; Sysolyatin, S. V.; Sakovich, G. V.; Surmacheva, I. A.; Surmachev, V. N.; Lapinab, Y. T. Russ. Chem. Bull. 2009, 58, 2164.

[23] Fotouhi-Far, F.; Bashiri, H.; Hamadanian, M. Propellants Explos., Pyrotech. 2017, 42, 213.

[24] Fotouhi-Far, F.; Bashiri, H.; Hamadanian, M.; Keshavarz, M. H. Inorg. Nano-Met. Chem. 2017, 47, 1489.

[25] Ji, H.-T.; Jing, Q.; Huang, J.-W.; Silverman, R. B. Tetrahedron 2012, 68, 1359.

[26] Zhu, S.-L.; Zhu, J.; Zhang, W.-P.; Cheng, C.; Shi, H.-J. Chin. J. Synth. Chem. 2014, 22, 664 (in Chinese). (朱思兰，朱晶；张伟萍，陈程，史海健，合成化学，2014，22， 664.)

[27] Hou, T.-J.; Zhang, J.; Wang, C.-J.; Luo, J. Org. Chem. Front. 2017, 4,1819 .

[28] Bailey, P. D.; Beard, M. A.; Dang, H. P. T.; Phillips, T. R.; Price, R. A.; Whittaker, J. H. Tetrahedron Lett. 2008, 49, 2150.

[29] Cheng, C.-J.; Sun, J.-W.; Xing, L.-X.; Xu, J.-M.; Wang, X.-Y.; Hu, Y.-F. J. Org. Chem. 2009, 74,5671.

[30] Quast, H.; Walter, S. J.; Mueller, B. Heterocycles 2012, 85, 2449.

[31] Gao, J.-H. M.S. Thesis, Zhejiang University of Technology, Hangzhou, 2012 (in Chinese) (高金华，硕士论文，浙江工业大学，杭州, 2012.)

[32] Zhang, M.-G.; Ma, Y.-Q.; Xia, Z.-J.; Chen, Z.-X. Chin. J. Pharm. 
2014, 45, 404 (in Chinese).

(张明光, 马堰启, 夏正君, 陈再新, 中国医药工业杂志, 2014, 45, 404.)

[33] Lu, D.-Q.; Wang, W.-B.; Ling, X.-Q.; Xie, J.; Shen, D. Mod. Chem. Ind. 2014, 34, 33 (in Chinese). (卢定强, 王维胞, 凌岫泉, 解杰, 沈东, 现代化工, 2014, 34, 33.)

[34] Yu, L.-D.; Xu, Q.-X.; Wang, X. J. Jiangxi Norm. Univ. (Nat. Sci. Ed.) 2017, 45, 507 (in Chinese).

(喻理德, 徐其雄, 王星, 江西师范大学学报 (自然科学版), 2017, 45, 507.)

[35] Désogère, P.; Rousselin, Y.; Poty, S.; Bernhard, C.; Goze, C.; Boschetti, F.; Denat, F. Eur. J. Org. Chem. 2014, 35, 7831.

[36] Torres, E.; Leiva, R.; Gazzarrini, S.; Carrizo, M. R.; Vivas, M. F.; Moroni, A.; Naesens, L.; Vázquez, S. ACS Med. Chem. Lett. 2014, 5,831 .

[37] Li, Y.; Manickam, G.; Ghoshal, A.; Subramaniam, P. Synth. Commun. 2006, 36, 925.

[38] Lou, D.-Y.; Wang, H.-S.; Liu, S.; Li, L.; Zhao, W.; Chen, X.-H.; Wang, J.-L.; Li, X.-Q.; Wu, P.-F.; Yang, J. Catal. Commun. 2018, 109, 29.

[39] Shu, C.; Zheng, C.-Z.; Wang, R.-J.; Zhang, J.-Y. Chemistry 2004, 67 (in Chinese) (舒畅，郑纯智，王日杰，张继炎，化学通报, 2004, 67.)

[40] Elamin, B.; Anantharamaiah, G. M.; Royer, G. P.; Means, G. E. J. Org. Chem. 1979, 44, 3442.

[41] Ram, S.; Spicer, L. D. Tetrahedron Lett. 1987, 28, 515.

[42] Anwer, M. K.; Spatola, A. F. Tetrahedron Lett. 1981, 22, 4369.

[43] Anwer, M. K.; Spatola, A. F.; Bossinger, C. D.; Flanigan, E.; Liu, R.-C.; Olsen, D. B.; Stevenson, D. J. Org. Chem. 1983, 48, 3503.

[44] Adger, B. M.; O'Farrell, C.; Lewis, N. J.; Mitchell, M. B. Synthesis 1987, 53.

[45] Khan, S. A.; Sivanandaiah, K. M. Synthesis 1978, 750.

[46] Felix, A. M.; Heimer, E. P.; Lambros, T. J.; Tzougraki, C.; Meienhofer, J. J. Org. Chem. 1978, 43, 21, 4194.

[47] Gray, B. D.; Jeffs, P. W. J. Chem. Soc., Chem. Commun. 1987, 18, 1329.

[48] Zheng, Y.; Wu, G.; Zhu, X. R.; Li, Y.-J.; Ma, Y.-H.; Zhao, X.; Lu, T.; Zhu, Y.-Q. Chem. Res. Chin. Univ. 2011, 27, 224

[49] Chen, S.-P.; Liu, D.-Q.; Si, L.-L.; Chen, L.-G.; Yan, X.-L. Synth. Commun. 2017, 47, 3, 238.

[50] Guo, M.-L.; Hong, K.-H.; Lv, Y.- F.; Ding, Y.; Li, C.-C.; Xu, H.; Qi, W.-X.; Chen, J.-Q.; Ji, M.; Cai, J. J. Chem. Res. 2017, 2, 112.

[51] Bajwa, J. S.; Slade, J.; Repic, O. Tetrahedron Lett. 2000, 41, 6025.

[52] Babu, S. N. N.; Srinivasa, G. R.; Santhosh, D. C.; Gowda, D. C. J. Chem. Res. 2004, 66.

[53] Babu, S. N. N.; Srinivasa, G. R.; Santhosh, D. C.; Gowda, D. C. Synth. Commun. 2004, 34, 1831.

[54] Kim, S. S.; Lin, G.; Yang, J. W. Bull. Korean Chem. Soc. 2004, 25, 249.

[55] Davies, S. G.; Mortimer, D. A. B.; Mulvaney, A. W.; Russell, A. J.; Skarphedinsson, H.; Smith, A. D.; Vickers, R. J. Org. Biomol. Chem. 2008, 6, 1625.

[56] Hungerhoff, B.; Samanta, S. S.; Roels, J.; Metz, P. Synlett 2000, 77.

[57] Oikawa, Y.; Yoshioka, T.; Yonemitsu, O. Tetrahedron Lett. 1982, 23,885 .

[58] Oikawa, Y.; Yoshioka, T.; Yonemitsu, O. Tetrahedron Lett. 1982, 23,889

[59] Singh, S. B. Tetrahedron Lett. 1995, 36, 2009.

[60] Yamaura, M.; Suzuki, T.; Hashimoto, H.; Yoshimura, J.; Okamoto, T.; Shin, C. M. Bull. Chem. Soc. Jpn. 1985, 58, 1413.

[61] Davies, S. G.; Ichihara, O. Tetrahedron Lett. 1998, 39, 6045.

[62] Bull, S. D.; Davies, S. G.; Fenton, G.; Mulvaney, A. W.; Prasad, R. S.; Smith, A. D. Chem. Commun. 2000, 337.

[63] Bull, S. D.; Davies, S. G.; Fenton, G; Mulvaney, A. W.; Prasad, R. S.; Smith, A. D. J. Chem. Soc., Perkin Trans. 1 2000, 3765.

[64] Chen, S.-S.; Qiu, W.-G.; Yu, Y.-Z. Chin. J. Explos. Propellants 2000, 2, 4 (in Chinese)

(陈树森，邱文革，于永忠，火炸药学报, 2000, 2, 4.)
[65] Qiu, W.-G.; Chen, S.-S; Yu, Y.-Z. Chin. J. Chem. 1999, 17, 554

[66] Gigg, R.; Conant, R. J. Chem. Soc. Chem. Commun. 1983, 465.

[67] Haddach, A. A.; Kelleman, A.; Deaton-Rewolinski, M. V. Tetrahedron Lett. 2002, 43, 399

[68] Kong, W.; Fu, C.; Ma, S. Chem.-Eur. J. 2011, 17, 13134.

[69] Semak, V.; Escolano, C.; Arroniz, C. Tetrahedron: Asymmetry 2010, $21,2542$.

[70] Zhu, W.-T.; Gan, H.-F.; Chen, Z.-B.; Guo, K. Synth. Chem. 2015, 23, 977 (in Chinese). (朱文通，甘海峰，陈正帮，郭凯，合成化学，2015, 23,977.)

[71] Page, P. C. B.; Stephenson, G. R.; Harvey, J.; Slawin, A. M. Z. Synlett 2016, 27, 2500

[72] Zhao, F.; Tian, W.-H.; Luo, F.; Cheng, H.-L.; Jiang, Y.-B.; Chen, Z Synth. Commun. 2016, 46, 20, 1678.

[73] Feula, A.; Fossey, J. S. RSC Adv. 2013, 3, 16, 5370

[74] Kroutil, J.; Trnka, T.; Cerny, M. Org. Lett. 2000, 2, 1681.

[75] Kroutil, J.; Trnka, T.; Cerny, M. Synthesis 2004, 446.

[76] Moriyama, K.; Nakamura, Y.; Togo, H. Org. Lett. 2014, 16, 3812

[77] Ghosh, S.; Shashidhar, J. Tetrahedron Lett. 2009, 50, 1177.

[78] Amat, M.; Pérez, M.; Minaglia, A. T.; Casamitjana, N.; Bosch, J. Org. Lett. 2005, 7, 3653.

[79] Amat, M.; Escolano, C.; Gómez-Esqué, A.; Lozano, O.; Llor, N.; Griera, R.; Bosch, J. Tetrahedron: Asymmetry 2006, 17, 1581.

[80] Torregrosa, R.; Pastor, I. M.; Yus, M. Tetrahedron 2007, 63, 947.

[81] Morales, C. L.; Pagenkopf, B. L. Org. Lett. 2008, 10, 157.

[82] Garst, M. E.; Dolby, L. J.; Esfandiari, S.; Fedoruk, N. A.; Chamberlain, N. C.; Avey, A. A. J. Org. Chem. 2000, 65, 7098.

[83] Suzuki, H.; Tsukuda, A.; Kondo, M.; Aizawa, M.; Senoo, Y; Nakajima, M.; Watanabe, T.; Yokoyama, Y.; Murakami, Y. Tetrahedron Lett. 1995, 36, 1671.

[84] Rao, T. S.; Pandey, P. S. Synth. Commun. 2004, 34, 3121.

[85] Franceschini, N.; Da N. S.; Miel, H.; Sonnet, P. Heterocycl. Commun. 2005, 11, 509.

[86] Franceschini, N.; Da N. S.; Miel, H.; Sonnet, P. Tetrahedron: Asymmetry 2003, 14, 3401 .

[87] Nandi, P.; Dye, J. L.; Jackson, J. E. Tetrahedron Lett. 2009, 50, 3864.

[88] Choudary, B.; Giles, R. G.; Jovic, F.; Lewis, N.; Moore, S.; Urquhart, M. Org. Process Res. Dev. 2012, 16, 1927.

[89] Shaw, A. Y.; Mclaren, J. A.; Nichol, G. S.; Hulme, C. Tetrahedron Lett. 2012, 53, 2592.

[90] Lee, S. H.; Mu, Y.; Kim, G. W.; Kim, J. S.; Park, S. H.; Jin, T.; Lee, K. Y.; Ham, W. H. Heterocycles 2013, 87, 1749.

[91] Lam, T. Y.; Wang, Y.-P.; Danheiser, R. L. J. Org. Chem. 2014, 45, 9396.

[92] Rombouts, F.; Franken, D.; Martinez-Lamenca, C.; Braeken, M.; Zavattaro, C.; Chen, J. S.; Trabanco, A. A. Tetrahedron Lett. 2010, $51,4815$.

[93] Lima, H. M.; Sivappa, R.; Yousufuddin, M.; Lovely, C. J. J. Org. Chem. 2014, 79, 2481.

[94] Chern, C. Y.; Huang, Y. P.; Kan, W. M. Tetrahedron Lett. 2003, 44, 1039.

[95] Yu, Y.-Z.; Guan, X.-P. Chin. J. Energ. Mater. 1999, 7, 1 (in Chinese). (于永忠, 管晓培, 含能材料, 1999, 7, 1.)

[96] Lv, L.-Y.; Ou, Y.-X.; Wang, J.-L.; Chen, B.-R. Fine Chem. 2003, 20, 577 (in Chinese). (吕连营，欧育湘，王建龙，陈博仁，精细化工, 2003, 20, 577.)

[97] Bayat, Y.; Hajimirsadeghi, S. S.; Pourmortazavi, S. M. Org. Process Res. Dev. 2011, 15, 810 .

[98] Bayat, Y.; Ebrahimi, H.; Fotouhi-Far, F. Org. Process Res. Dev. 2012, 16, 1733.

[99] Bayat, Y.; Hajighasemali, F. Propellants Explos. Pyrotech. 2016, $41,893$.

[100] Ou, Y.-X.; Liu, J.-Q. High Energy Density Compound, National Defence Industrial Press, Beijing, 2005, pp. 96 110 (in Chinese). (欧育湘, 刘进全, 高能量密度化合物, 国防工业出版社, 北京, 2005, pp. 96 110.) 
[101] Yang, B. W.; O'Rourke D, J. L. I. Synlett 1993, 195.

[102] See, Y. B. JP 321962, 1994.

[103] Wright, W. B.; Brabander, H. J. J. Org. Chem. 1961, 26, 10

[104] Olofson, R. A.; Martz, J. T. Senet, J. P.; Piteau, M.; Malfroot, T. J. Org. Chem. 1984, 49, 2081.

[105] Kanth, J. V. B,; Reddy, C. K.; Periasamy, M. Synth. Commun. 1994, 24,313

[106] Igarashi, J.; Kobayashi, Y. Tetrahedron Lett. 2005, 46, 6381.

[107] Annamalai, M.; Hristeva, S.; Bielska, M.; Ortega, R.; Kumar, K. Molecules 2017, 22, 827.

[108] Pandey, G.;. Rani, K. S. Tetrahedron Lett. 1988, 29, 4157.

[109] Smith, A. J.; Young, A.; Rohrbach, S.; O'Connor, E. F.; Allison, M.;
Wang, H.-S.; Poole, D. L.; Tuttle, T.; Murphy, J. A. Angew. Chem., Int. Ed. 2017, 56, 13747.

[110] Golantsov, N. E.; Karchava, A. V.; Yurovskaya, M. A. Chem. Heterocycl. Compd. 2006, 42, 1021.

[111] Montero, M. L.; Rodríguez, D. A.; Gotor, F. V.; Fernández, V. G.; Lavandera, I. Green Chem. 2015, 46, 2794.

[112] Grayson, E, J.; Davis, B. G. Org. Lett. 2005, 7, 2361.

[113] Baker, S. R.; Parsons A. F.; Wilson M. Tetrahedron Lett. 1998, 39, 331.

[114] Yanada, R.; Obika, S.; Kobayashi Y.; Inokuma, T.; Oyama, M.; Yanada, K.; Takemoto Y. Adv. Synth. Catal. 2005, 347, 1632. 